\title{
Calculation of IMAGE/MENA geometric factors and conversion of images to units of integral and differential flux
}

\author{
M. G. Henderson, a) M. F. Thomsen, R. Skoug, M. H. Denton, \\ R. Harper, and H. O. Funsten \\ ISR-1, Los Alamos National Laboratory, Los Alamos, New Mexico 87545 \\ C. J. Pollock \\ Southwest Research Institute, San Antonio, Texas
}

(Received 13 October 2004; accepted 1 February 2005; published online 1 April 2005)

\begin{abstract}
The Medium Energy Neutral Atom (MENA) instrument flown on the NASA IMAGE spacecraft is a time-of-flight neutral particle imager designed to image energetic neutral atom emissions from the Earth's inner magnetosphere over an energy per mass range of 1-60 keV/amu. Images are generated by combining data from three separate heads and have a nominal angular resolution of $4^{\circ} \times 4^{\circ}$. Here, we present a first-principles calculation of the geometric factors for each of the start-byte/stop-byte combinations for each of the three heads in the IMAGE/MENA instrument based on a detailed understanding of the its physical construction. The geometric factors are used to compute combined integral flux images and it is demonstrated that they result in head-to-head matching of the data that are both continuous and physically reasonable. We also discuss several issues associated with energy binning as a means for constructing differential flux images and present a powerful and robust approach that solves several critical problems inherent with this type of instrument. (C) 2005 American Institute of Physics. [DOI: 10.1063/1.1884190]
\end{abstract}

\section{INTRODUCTION}

The Medium Energy Neutral Atom (MENA) instrument on the IMAGE spacecraft ${ }^{1}$ was designed to image the energetic neutral atom (ENA) emissions from the earth's magnetosphere over an energy-per mass range of $1-60 \mathrm{keV} / \mathrm{amu}$ with a nominal angular resolution of $4^{\circ} \times 4^{\circ}$. The instrument is comprised of a set of three (nominally) identical sensor "heads" with head 2 pointing perpendicular to the spacecraft spin axis and heads 3 and 1 offset by $\pm 20^{\circ}$ with respect to the perpendicular direction. Photographs of the MENA instrument mounted on the IMAGE spacecraft and also in the laboratory during calibration are shown in Figs. 1(a) and 1(b). In panel (a), the spin axis of the spacecraft points toward the top of the page, and the MENA instrument is marked with an arrow. From left to right the heads are identified as: head 3, head 2, and head 1 . The heads image only in the polar angle direction (with respect to the spin axis) and are collimated in the azimuthal direction [the transmission probability in the azimuthal direction has a full width at half maximum (FWHM) of approximately $\pm 4^{\circ}$ ]. Imaging in the azimuthal direction is facilitated by the rotation of the spacecraft (the spin period is nominally $120 \mathrm{~s}$ ). Three heads are required in order to increase the total field of view (FOV) in the polar angle direction and in order to fill in the central gaps in coverage in each head.

A schematic illustration of the imaging concept for a single head is shown in Fig. 1(d). Incident ENAs are initially collimated in azimuth by a set of 21 curved parallel plates.

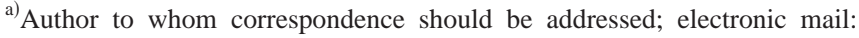
mghenderson@lanl.gov
}

These plates are alternately biased with high voltage (on the order of $3 \mathrm{kV}$ ) in order to sweep out ions with energies up to about $20 \mathrm{keV}$. An ENA must then pass through a freestanding transmission grating nano-structure designed to transmit heavy particles but reject UV photons. Following this, the ENA passes through a thin carbon foil (mounted on the bottom side of the grating structure). ENAs exiting the foil generate secondary electrons that we call "start electrons." These electrons are accelerated by a grid which causes them to move very rapidly downward to the microchannel plate (MCP) detector in a $z$-stack configuration that subsequently generates a "start pulse" on a "start" position-sensitive anode. Meanwhile, the ENA continues on in a straight line path to impact the MCP detector and generates a "stop" pulse on the stop position-sensitive anode. Although they are mounted on a single board, the start and stop anodes are physically separated and electrically isolated, each having its own processing electronics. The stop anode lies on both sides of the start anode, and the two halves of this split anode are electrically coupled. The total variation in gain across the MCP is $<10 \%$. ${ }^{1}$

The quantities that are measured and reported for the detection of and ENA are: start position, stop position, start pulse height, stop pulse height, time of flight (TOF), head identification (ID), and the $4^{\circ}$-wide azimuthal sector in which the event occurred. The TOF is measured as the time difference between the start pulse and the stop pulse. On board the spacecraft, these quantities are all described using 8 bit words and the on-board image processing can take advantage of the full digital resolution. However, the directevents data that get telemetered to the ground have a reduced resolution. In the direct events data or "statistics data," the 

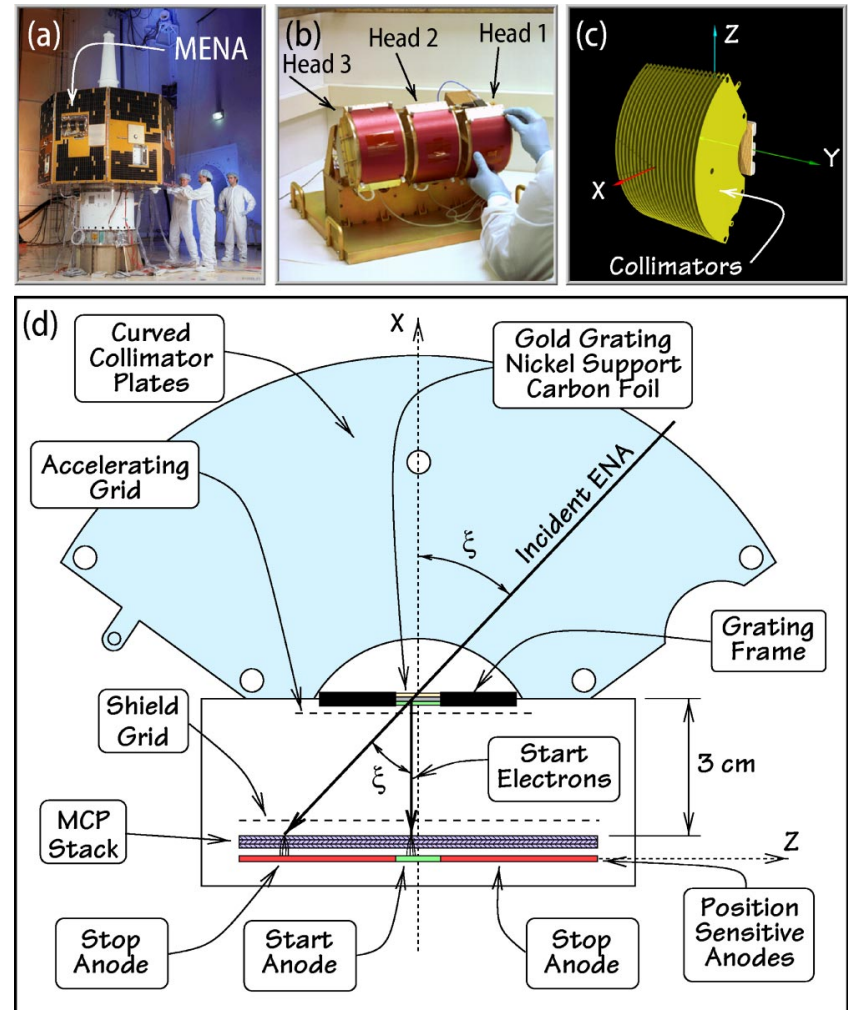

FIG. 1. (Color online) Overview of the IMAGE spacecraft and the MENA instrument. (a) The MENA instrument mounted on the IMAGE spacecraft. The spin axis is vertically upward in this photograph. (b) The MENA instrument in the laboratory with protective covers in place. (c) Schematic illustration of the MENA collimators and transmission grating assembly relative to the coordinate system used throughout the article. (d) Schematic illustration of a single MENA head. The various components shown in (c) and (d) are not to scale.

byte values are down sampled as shown in Table I. A further complication is that, due to telemetry constraints, only a subset of the measured direct events can be included in the telemetered direct events data set (typically we receive no more than $\approx 13000$ events per spin on the ground).

For the azimuth bin, the direct events data contain a 6 bit coarse resolution word specifying an $8^{\circ}$-wide azimuth bin, plus a 1 bit word indicating whether the event occurred in the lower $4^{\circ}$ portion of the $8^{\circ}$ bin or the upper $4^{\circ}$ portion of the $8^{\circ}$ bin. However, since the size of the telemetry buffer allocated for MENA typically allows for the storage of no more than $\approx 13000$ direct events (per spin) there is a limited number of events that can be reported back to the ground in one spin of the spacecraft. Thus the quota of reported events is usually spent on those that occurred in the lower half of the coarse bin. This unfortunately limits the azimuthal resolution of the direct events data to just $8^{\circ}$ or 45 bins per spin as indicated by the bracketed quantity in Table I.

In addition to the direct events data, we also receive on the ground the total singles counts for start, stop, and valid (coincidence) events in each head accumulated over an entire spin. These quantities allow us to scale the direct event count rates up to what was observed on board-although this scaling process does not compensate for the poorer counting statistics inherent in the direct-events data.

Before launch it was anticipated that the measured quan-
TABLE I. Hardware byte down sampling.

\begin{tabular}{ccccc}
\hline \hline & \multicolumn{2}{c}{ On board } & \multicolumn{2}{c}{ Direct events } \\
\cline { 2 - 5 } Digitized quantity & No. bits & No. vals & No. bits & No. vals \\
\hline Start position & 8 & 256 & 4 & 16 \\
Stop position & 8 & 256 & 7 & 128 \\
Start height & 8 & 256 & 6 & 64 \\
Stop height & 8 & 256 & 6 & 64 \\
TOF & 8 & 256 & 6 & 64 \\
Head ID & 8 & 3 & 2 & 3 \\
Azimuth bin & 8 & 90 & $6+1$ & 90 (45 \\
& & & & effective bins) \\
\hline \hline
\end{tabular}

tities would be used as follows. First, the start and stop positions would be used to determine the incident angle, $\xi$. The TOF and incident angle would be used to determine the speed. The pulse heights were then supposed to allow for resolution of mass (e.g., $\mathrm{H}$ or $\mathrm{O}$ ). And finally the energy would be determined from the mass and speed. In practice, this scheme works very well except that so far, we have not been successful in using the pulse heights to extract species information. Thus, for the time being we assume that all the ENAs are hydrogen. However, as we shall see later in this document, there is still a chance that we may yet be able to separate hydrogen and oxygen in the direct-events data.

A much more detailed description of the MENA instrument can be found elsewhere. ${ }^{1}$ In this document, we focus specifically on the process of converting the raw directevents data into images with units of integral and differential flux. In doing so, we present a first-principles calculation of the MENA geometric factors based on a detailed understanding of the physical design of the instrument. Following this, we discuss issues associated with energy binning and we present a solution that not only overcomes these issues, but also offers the possibility of extracting species information as well.

\section{CONVERTING FLUX TO COUNTS}

Following the formalism of Sullivan, ${ }^{2}$ the coincidence counting rate obtained with a particle telescope can be written

$$
\begin{aligned}
C= & \frac{1}{T} \int_{t_{0}}^{t_{0}+T} \int_{S} \int_{\Omega} \int_{E} \\
& \times \sum_{s} \tau_{s}(E, \sigma, \hat{u}, t) j_{s}(E, \hat{u}, t) \hat{u} \cdot d \boldsymbol{\sigma} d t d \omega d E
\end{aligned}
$$

where,

$$
\begin{aligned}
C= & \text { coincidence counting rate }\left(s^{-1}\right) ; \\
T= & \text { total observing time }(s) ; \\
t_{0}= & \text { time at start observation, } \\
s= & \text { species label, } \\
\tau_{s}= & \text { overall detection efficiency for particles of } \\
& \text { species } s . \text { It can very as a function of energy, } \\
& \text { look direction detection location and time; } \\
j_{s}= & \text { incident differential flux for species } s \\
& \left(\mathrm{~cm}^{-2} \mathrm{sr}^{-1} \mathrm{keV}^{-1} \mathrm{~s}^{-1}\right) ; \\
\hat{u}= & \text { look direction; }
\end{aligned}
$$




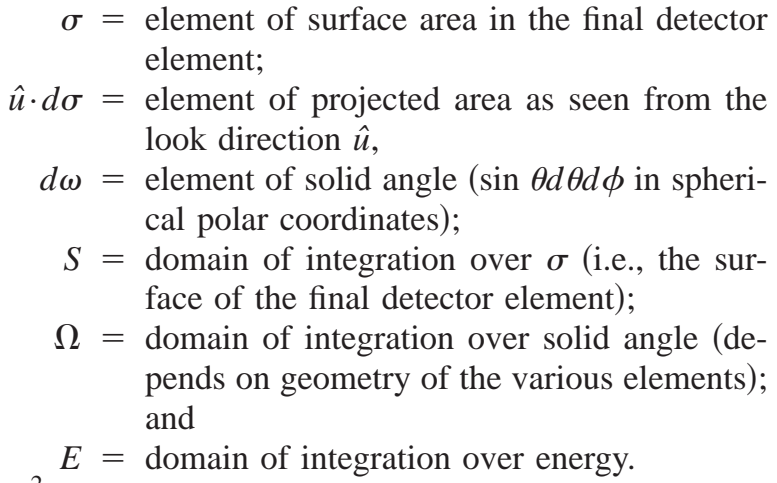

Sullivan $^{2}$ used this expression analytically for straight line particle trajectories that make it through the apertures to the detector. However, it can also be evaluated (e.g., via Monte Carlo integration) for more complex trajectories (e.g., curved trajectories of charged particles in a magnetic field). In addition, Tuszewski, Cayton, and Ingraham ${ }^{3}$ have recently extended Sullivan's formalism to analytically include more complicated particle trajectories that can contribute to the count rate despite the fact that they do not necessarily pass through the geometrical apertures. This newer "analytical probability" (AP) approach can account for features such as penetrating particles, scattering, reflections and secondary bremsstrahlung photons. Although in future work we may need to include some of these more complicated effects, here we will restrict our calculations to simple straight-line trajectories that have a clear geometrical path through the aperture and adopt Sullivan's original formalism.

In order to greatly simplify the above expression, we assume: (1) only a single species is present (or we can separate multiple species); (2) the detection efficiency does not vary with position on the detector; (3) the detection efficiency is constant over the integration time; (4) the incident flux does not change over the integration time, and; (5) the energy and angular dependencies of the incident flux are separable such that $j(E, \theta, \phi)=j_{0}(E) F(\theta, \phi)$. Then (in spherical polar coordinates), we can rewrite Eq. (1) in terms of a "directional response function," $R(E, \theta, \phi)$ and an "instrument gathering power," $\Gamma(E)$.

The directional response function (or "effective area" as it is often called) is defined as

$$
\begin{aligned}
R(E, \theta, \phi) & =\tau(E, \theta, \phi) \int_{S} \hat{u} \cdot d \boldsymbol{\sigma} \\
& =\tau(E, \theta, \phi) A(\theta, \phi),
\end{aligned}
$$

where $A(\theta, \phi)$ is the projected area in the direction $(\theta, \phi)$ and $\tau(E, \theta, \phi)$ can be thought of as an additional direction and energy dependent probability for particles to get to the detector (e.g., due to the collimating structures and start foil in our case). The instrument gathering power is defined as

$$
\Gamma(E)=\int_{\theta} \int_{\phi} R(E, \theta, \phi) F(\theta, \phi) \sin \theta d \theta d \phi .
$$

Thus, Eq. (1) can be rewritten as

$$
C=\int_{E} j_{0}(E) \Gamma(E) d E .
$$

Note that if the incident flux is constant over the angular range of integration, we can set $F(\theta, \phi)=1$. The gathering power then becomes the familiar "geometric factor," $G$ given by

$$
G(E)=\int_{\theta} \int_{\phi} R(E, \theta, \phi) \sin \theta d \theta d \phi .
$$

Furthermore, if $j(E)$ is approximately constant over the energy integration range, we have the familiar flux conversion formula

$$
C=j_{0}(E) G(E) \Delta E
$$

or

$$
j_{0}(E)=\frac{C}{G(E) \Delta E} .
$$

As we shall see later, the generation of differential flux images from the direct events data is not quite as simple as applying this formula to every pixel, but we will still need to compute fluxes with it.

In order to proceed with the calculation of the geometric factors, it is crucially important to recognize what coincidence measurements we are actually making with the MENA instrument. Unlike more conventional particle telescopes, the MENA instrument is inherently an imaging device. Thus, we are not just interested in the fact that a neutral atom passed through the entrance aperture and hit the detector-that is not the measurement that we are after. Instead, we are interested in making a large number of individual measurements of the following sort: a particle passed through a small portion of the entrance aperture and landed in a small portion of the detector. In other words, we are making measurements of the count rate observed when a particle has a specific startbyte value and a specific stop-byte value. A single head can then be thought of as one that is comprised of (on the order of) $16 \times 128$ individual little particle telescopes-as many as 16 start-byte "apertures" and as many as 128 stop-byte "detectors." In practice, for MENA, only ten start-byte values map to physical regions in the entrance aperture. And there are also substantially fewer than 128 "valid" stop-byte values possible due to the fact that the central portion of the position sensitive anode is dedicated to measuring start events.

Another way to think about this is to imagine a somewhat differently designed instrument. Let us suppose that the entrance aperture really was a narrow slit and the detector plane was comprised of an array of 128 discrete silicon detectors. Then it is easy to see that each aperture/detector pair comprises a separate particle telescope. This type of design is in fact implemented in the Polar CEPPAD/inverse photoemission spectroscopy (IPS) instrument ${ }^{4}$ on a much smaller scale. In IPS, there are three collimated heads and each head is comprised of a slit camera with three discrete silicon detectors. With MENA, we essentially have ten of these slit detectors stacked next to each other by virtue of the fact that the start-byte value tells us the "subaperture" through which the particle entered the instrument. In essence, the MENA 

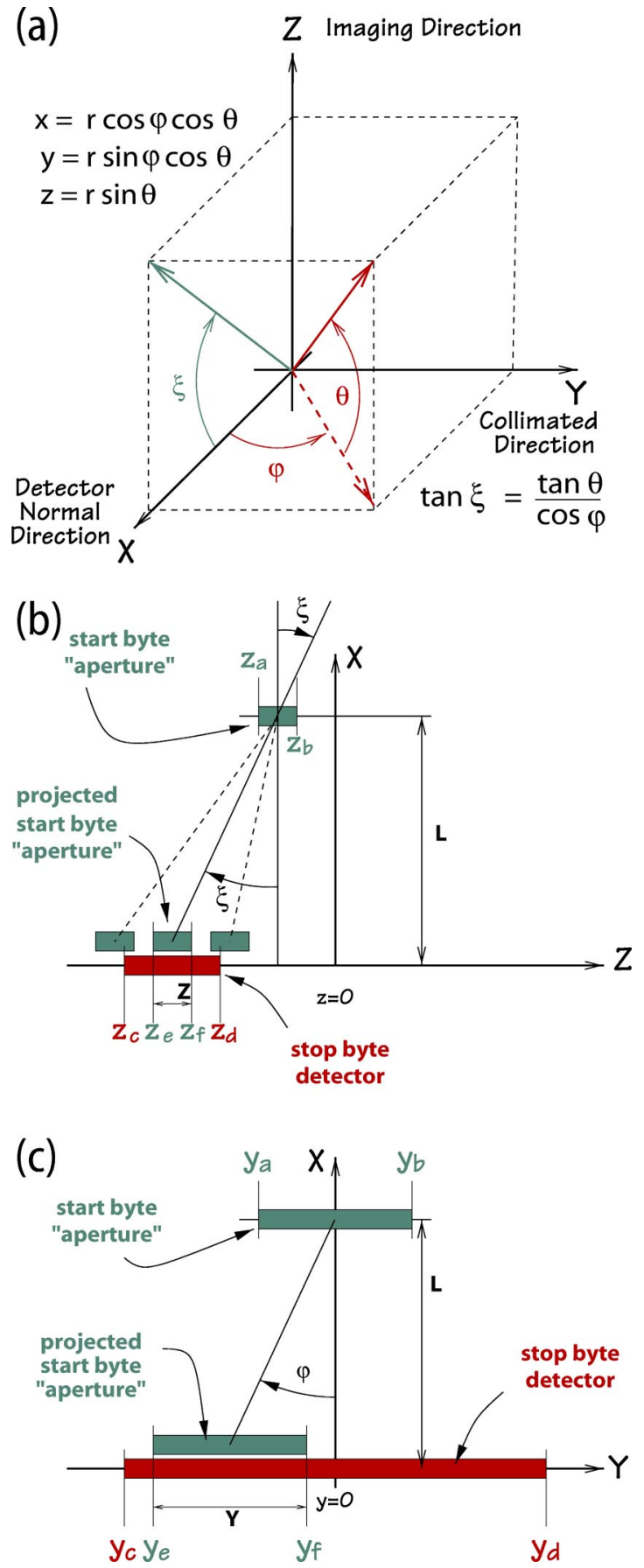

FIG. 2. (Color online) (a) Definition of coordinate system used to compute the projected area. (b) Schematic showing area overlap in the $z$ or "imaging" direction. The start-byte aperture is shown projected down to the detector plane at three different angles. The left and right cases illustrate partial overlap while the central case illustrates complete overlap. As $\xi$ increases, the area of overlap will first increase linearly from zero to a maximum "plateau" value and then decrease linearly from the maximum value back to zero. [E.g., see Fig. 4(b).] (c) Area overlap in the $y$ or "collimated" direction. In (b) and (c), the relative sizes of the start and stop regions are not to scale.

design is the same as the IPS design except that both the physical location of the entrance aperture and the physical bounds of each detector are electronically selected-and this electronic selection is accomplished via the start- and stopbyte assignments.

In this interpretation of the MENA instrument, the specific start-byte value corresponds to a physical aperture
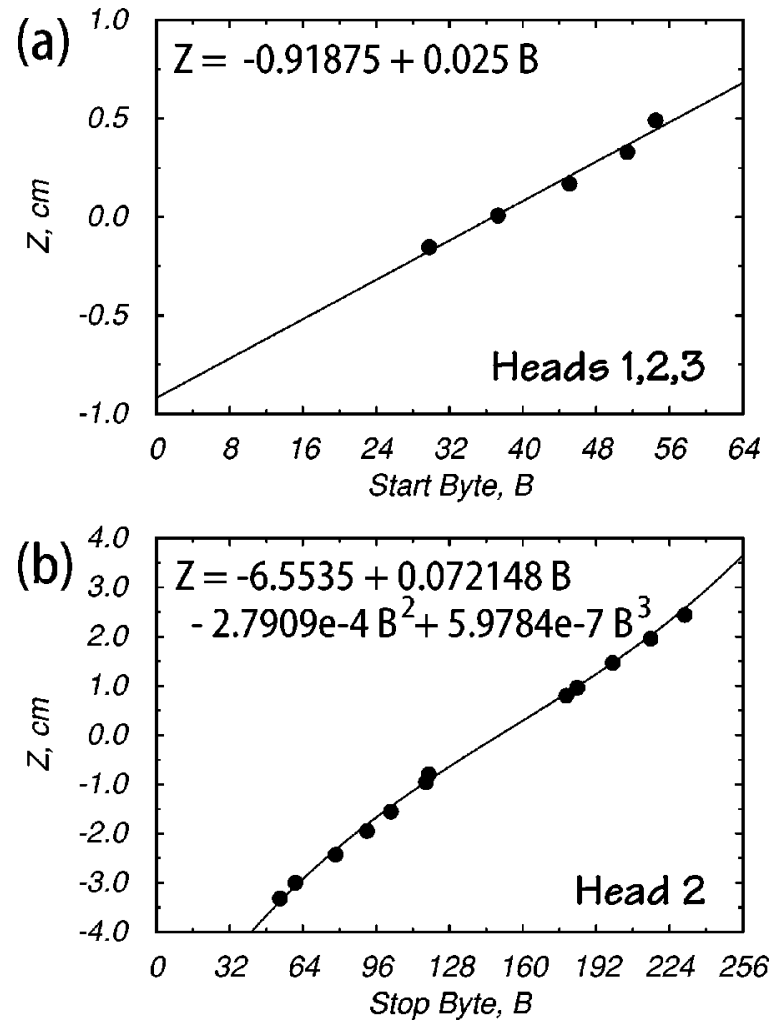

FIG. 3. Calibration measurements of the start byte and stop byte to position mappings. The straight line fit to the start position vs start byte curve shown in (a) was used for all three heads. Head-dependent cubic polynomials were used to fit the stop position vs stop byte data. The cubic polynomial fit for head 2 is shown in (b).

width and the specific stop-byte value corresponds to a physical area on the detector. Thus, with knowledge about how the start and stop-byte values map to physical space, we can compute the projected area of each subdetector. This is exactly what is done in the following section.

\section{PROJECTED AREA FOR A START-BYTE/STOP- BYTE PAIR}

The projected area, $A(\theta, \phi)$, for a two-element telescope is easily computed using the so-called "shadow area" method (e.g., Sullivan ${ }^{2}$ ). In spherical-polar coordinates [Fig. 2(a)], $A(\theta, \phi)$ is given by

$$
A(\theta, \phi)=\mathbf{A}_{\text {shadow }}(\theta, \phi) \cdot \hat{u},
$$

where, $\mathbf{A}_{\text {shadow }}(\theta, \phi)$ is the overlapping surface area that results when the aperture is parallel projected down to the detector (i.e., it can be thought of as the area of the "shadow" that the aperture casts on the detector), and $\hat{u}$ $=(\cos \theta \cos \phi, \cos \theta \sin \phi, \sin \theta)$ is the direction the response is computed for. If the aperture and detector planes are in the $y-z$ plane then $\mathbf{A}_{\text {shadow }}(\theta, \phi)=\left(A_{s}, 0,0\right)$ so that

$$
A(\theta, \phi)=A_{s}(\theta, \phi) \cos \phi \cos \theta .
$$

Although Sullivan ${ }^{2}$ gives $A(\theta, \phi)$ for a two-element detector with different-sized rectangular apertures, the geometry is valid only for elements that are centered on the $x$ axis. Since we wish to compute the geometric factor for each and every start-byte/stop-byte combination, we need to compute the di- 

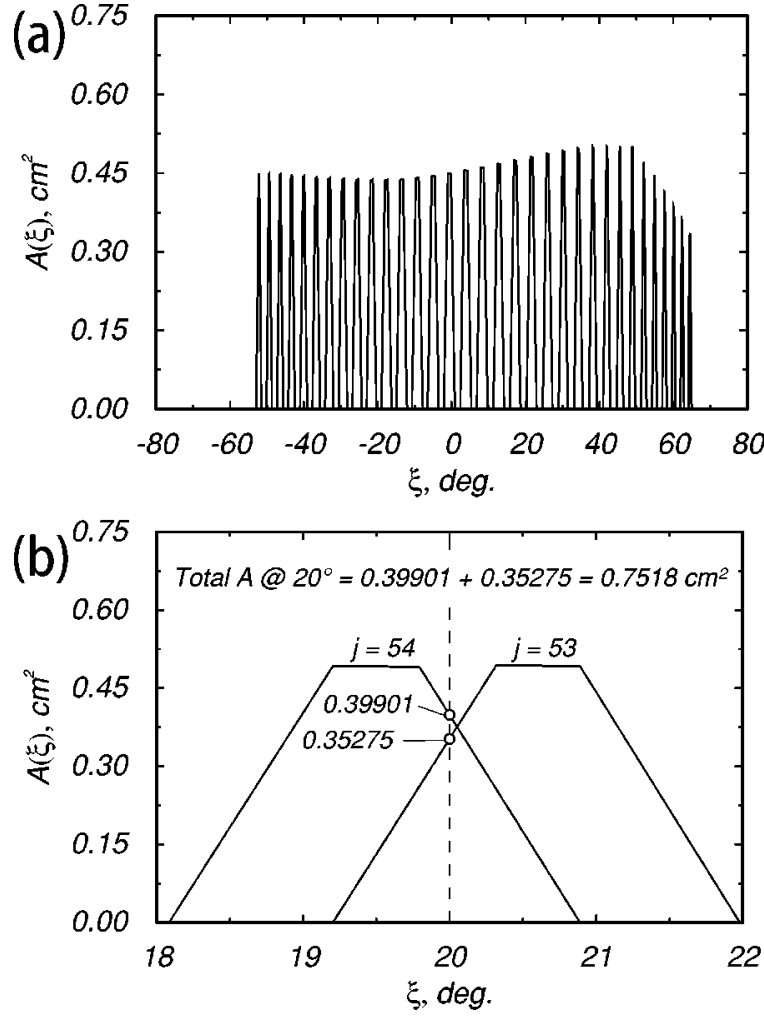

FIG. 4. Projected area [i.e., expression (18)] vs $\xi$ for $\phi=0^{\circ}$ and a start-byte value of 7 in head 2. (a) Curves for every fourth stop byte from 0 to 128 have been over plotted. (b) A zoomed in view for stop bytes 53 and 54 only. Note that constant, flat-topped plateaus occur in the shadow area, $A_{s}$, vs $\xi$ profiles whenever the parallel-projected start-byte aperture and the stop-byte "detector" area have different sizes. This combined with the trigonometric factors in expression (18) give rise to the variability in the upper envelope in (a). Note that the variability can be fairly complicated because over some angular ranges the start width is less than the stop width while for other angular ranges the reverse is true.

rectional response function for two rectangular elements that can be placed "off axis."

In Fig. 2(a), we present the coordinate system in which we shall compute $A(\theta, \phi)$. The $\theta$ and $\phi$ angles are the spherical-polar coordinates (note that $\theta$ is the latitude instead of the co-latitude) and $\xi$ is the angle between the $x$ axis and the projection of the three-dimensional (3D) vector onto the $x-z$ plane. Also shown is the relationship between $\xi, \theta$, and $\phi$. Figure 1(c) illustrates how a MENA head is oriented relative to this coordinate system.

The overlap in the $z$ direction (i.e., the imaging direction) is shown schematically in Fig. 2(b). For a given start byte, the detector is like a slit camera. And if we consider also only a single stop byte, then the detector has just the width of that stop byte. The values of $z_{a}, z_{b}, z_{c}, z_{d}$ have been determined experimentally for each start byte and stop byte and can be computed via empirically determined polynomials. The quantities $z_{e}$ and $z_{f}$ give the start-byte position projected down to the MCP at an angle of $\xi$. Thus

$$
z_{e}=z_{a}-L \tan \xi=z_{a}-L \frac{\tan \theta}{\cos \phi}
$$

$$
z_{f}=z_{b}-L \tan \xi=z_{b}-L \frac{\tan \theta}{\cos \phi} .
$$

If we define the quantities

$$
\begin{aligned}
& z_{1}=\max \left(z_{c}, z_{e}\right), \\
& z_{2}=\min \left(z_{d}, z_{f}\right)
\end{aligned}
$$

then the overlap distance is given by

$$
Z=\max \left(z_{2}-z_{1}, 0\right) \text {. }
$$

Figure 2(c) shows the geometry in the $y$ direction (i.e., collimated direction) and proceeding as above, we have

$$
\begin{aligned}
& y_{e}=y_{a}-L \tan \phi, \\
& y_{f}=y_{b}-L \tan \phi
\end{aligned}
$$

and

$$
\begin{aligned}
& y_{1}=\max \left(y_{c}, y_{e}\right), \\
& y_{2}=\min \left(y_{d}, y_{f}\right),
\end{aligned}
$$

which gives the following for the overlap distance in the $y$ direction:

$Y=\max \left(y_{2}-y_{1}, 0\right)$.

The shadow area (as a vector quantity) is then given as

$$
A_{\text {shadow }}=(Y Z, 0,0) \text {. }
$$

Finally, with, $\hat{u}=(\cos \theta \cos \phi, \cos \theta \sin \phi, \sin \theta)$, the projected area is given by

$$
A_{i j k}(\theta, \phi)=A_{\text {shadow }} \cdot \hat{u}=Y Z \cos \phi \cos \theta,
$$

where the subscripts $i, j$, and $k$ refer to individual start byte, stop byte, and head values. Note that since the empirically determined mapping from bytes to position (for both start and stop bytes) is different for each of the heads, the $A_{i j k}$ will in general all be somewhat different.

In order to proceed with the calculations outlined above, we must know the physical values of $z_{a}, z_{b}, z_{c}, z_{d}, y_{a}, y_{b}, y_{c}$, and $y_{d}$ for each of the start and stop-byte combinations for all three heads. The mappings from start and stop bytes to physical position along the anode have been determined from calibration data. The start-byte-to-position mappings were fit with straight lines for all heads as shown in Fig. 3(a), and the stop-byte-to-position mappings were fit with cubic polynomials as shown (for head 2) in Fig. 3(b). The position versus byte polynomials adopted for each head are as follows:

\section{Head 1}

$$
\begin{aligned}
Z_{\text {start }}= & -0.91875+0.025 B, \\
Z_{\text {stop }}= & -6.0695+0.064491 B-2.1694 \mathrm{e}-4 B^{2} \\
& +4.9283 \mathrm{e}-7 B^{3} .
\end{aligned}
$$

Head 2

$Z_{\text {start }}=-0.91875+0.025 B$, 


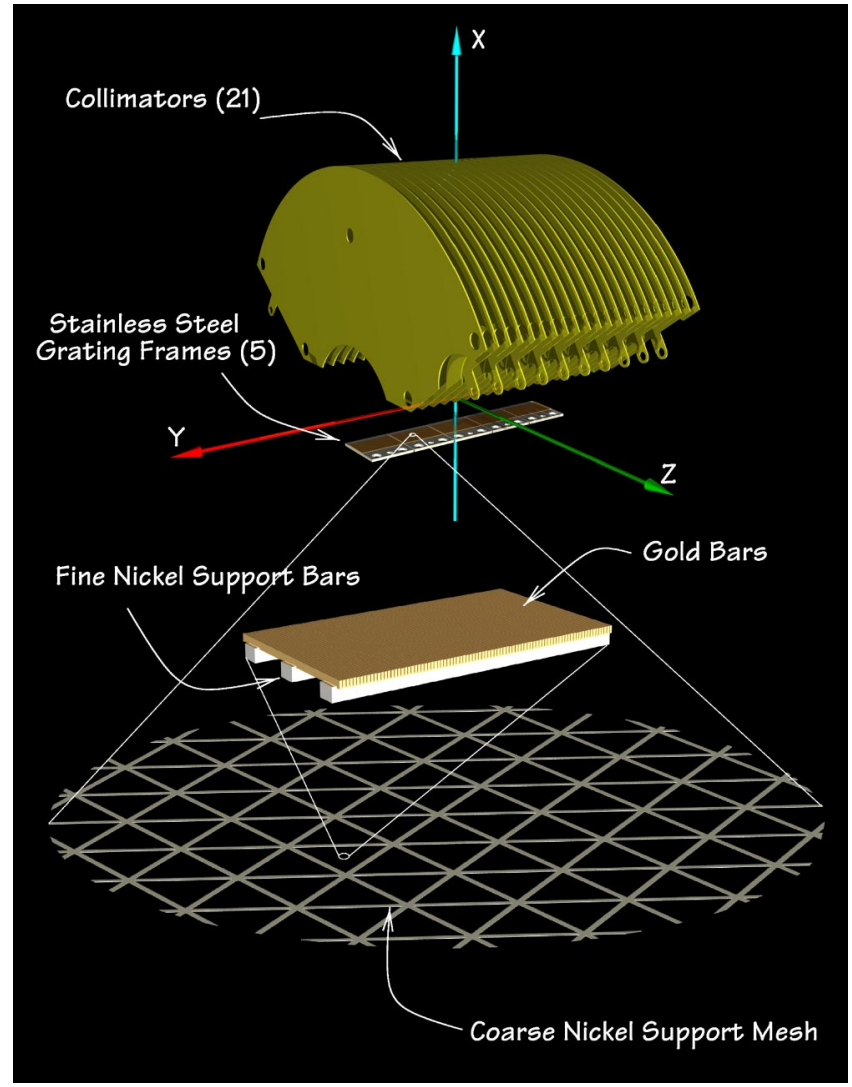

FIG. 5. (Color online) Exploded view of the MENA collimators and transmission gratings. Shown are the curved collimator plates, the coarse nickel support mesh, the gold bars and the fine nickel support grid. Note that the coarse mesh is the bottom-most layer with the nickel and gold grating structure resting on top of it. Figure is not to scale.

$$
\begin{aligned}
Z_{\text {stop }}= & -6.5535+0.072148 B-2.7909 \mathrm{e}-4 B^{2} \\
& +5.9784 \mathrm{e}-7 B^{3} .
\end{aligned}
$$

\section{Head 3}

$$
\begin{aligned}
Z_{\text {start }}= & -0.91875+0.025 B, \\
Z_{\text {stop }}= & -6.3115+0.0683195 B-2.48015 \mathrm{e}-4 B^{2} \\
& +5.45335 \mathrm{e}-7 B^{3} .
\end{aligned}
$$

For the purposes of deriving byte-to-position mappings, the calibration data for head 3 were not usable due to hardware problems at the time of calibration. Although in the future, we may be able to recover some additional information from the calibration data and/or utilize on-orbit data acquired from all three heads to place constraints on the head 3 mappings, this is a complicated task and is beyond the scope of the present article. Therefore, for our present purposes, the stop-byte-to-position polynomial for head 3 was derived by averaging the coefficients from heads 1 and 2 .

The above polynomials were initially used to derive the start/stop byte-to-angle mappings for both the on-board and statistics data. They are still used for the on-board data, but

\begin{tabular}{|c|c|c|c|c|}
\hline & Lot No. & $\begin{array}{c}\text { Wafer } \\
\text { ID }\end{array}$ & $\begin{array}{c}\text { Scribe } \\
\text { ID }\end{array}$ & $\begin{array}{c}\text { Frame } \\
\text { ID }\end{array}$ \\
\hline \multirow{5}{*}{ Head 1} & U04 & U04H010 & 3179 & IB037 \\
\hline & U02 & U02H021 & 2566 & IB057 \\
\hline & U04 & U04H006 & 3047 & IB062 \\
\hline & U04 & U04H004 & 3034 & IB074 \\
\hline & U04 & U04H021 & 3107 & IB083 \\
\hline \multirow{5}{*}{ Head 2} & U02 & U02H004 & 2693 & IB049 \\
\hline & U04 & U04H007 & 3152 & IB073 \\
\hline & U04 & U04H003 & 3151 & IB076 \\
\hline & U04 & U04H001 & 3158 & IB096 \\
\hline & U04 & U04H002 & 3188 & IB097 \\
\hline \multirow{5}{*}{ Head 3} & U04 & U04H007 & 3152 & IB029 \\
\hline & U02 & U02H004 & 2693 & IB038 \\
\hline & U04 & U04H008 & 3083 & IB075 \\
\hline & U04 & U04H001 & 3158 & IB089 \\
\hline & U04 & U04H002 & 3188 & IB 100 \\
\hline
\end{tabular}
for the statistics processing we have adopted new angle look up tables (LUTs) that were re-derived from the calibration data in a different manner.
TABLE II. MENA flight unit transmission gratings

Figure 4 illustrates the projected area obtained for a start byte of 7 in head 2 at $\phi=0^{\circ}$. In Fig. 4(a), the projected area for every fourth stop byte is over plotted while Fig. 4(b) shows more detail for only stop bytes 53 and 54 (which peak near $\xi=+20^{\circ}$ ).

\section{COLLIMATOR TRANSMISSION PROBABILITY}

In the previous section, we computed the directional response function for a two-element detector comprised of a single narrow start-byte aperture and a single narrow stopbyte detector. This would be sufficient for the MENA instrument if there were no collimator, no transmission gratings and no grating support structures. In reality, particles must get through all of those additional apertures before they reach the "start-byte aperture" on the foil. In order to deal with this further complication, we introduce a transmission function that specifies, for a given direction, the probability that a particle will reach the foil.

In this section we examine the types of collimating structures found in the IMAGE/MENA collimator/ transmission grating assembly and we determine, analytically, the separate transmission probability functions associated with each. These probability transmission functions are then combined together in order to obtain the overall transmission probability.

\section{A. Collimating structures in MENA}

A schematic 3D illustration of the relevant collimating structures in the MENA instrument is given in Fig. 5. Shown in the upper part of the figure are the 21 curved collimator plates and the five stainless steel frames holding the transmission gratings. In the lower part of the figure, enlarged views of the coarse triangular nickel support mesh, the fine nickel support bars, and the gold bars of the transmission grating are shown.

The fabrication of the transmission gratings is an extremely difficult process because the gold bars have to be 

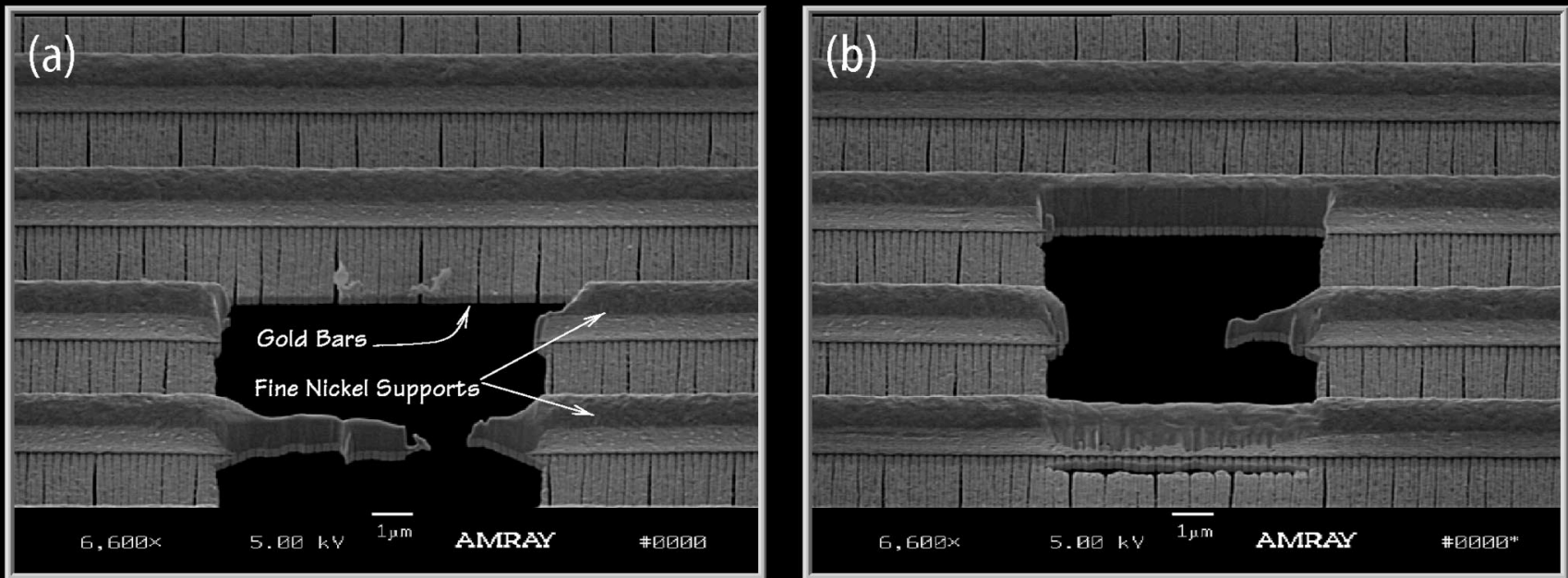

Dimensions of Fine Nickel Support Bars
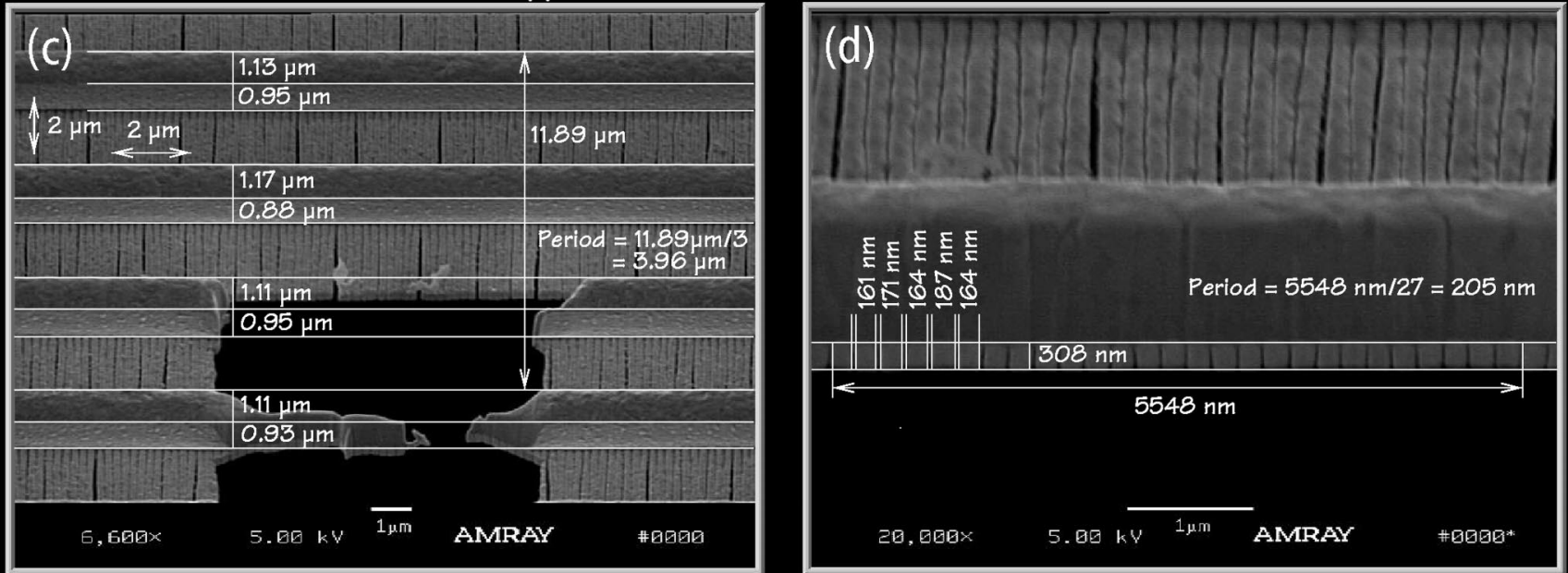

$W_{\text {avg }}=1.13 \mu \mathrm{m} \quad H_{\text {avg }}=0.93 \mu \mathrm{m} \quad P_{\text {avg }}=3.96 \mu \mathrm{m}$

$H=308 \mathrm{~nm} \quad P=205 \mathrm{~nm} \quad W a v g=169 \mathrm{~nm} \quad D=36 \mathrm{~nm}$

FIG. 6. Scanning electron micrographs (SEMs) of a nonflight-unit MENA transmission grating. (a) and (b) The gold bars are the vertically aligned structures and the fine nickel supports are the much larger horizontal bars. The rectangular holes were intentionally cut with an ion beam so that the dimensions of the bars could be measured. (c) Same as (a) but with dimensions overlayed. (d) A magnified view of the gold bars near the upper edge of the hole shown in (b).

separated by very small distances (on the order of $30-60 \mathrm{~nm}$ ) in order to achieve the desired UV-rejection and ion transmission characteristics. As a result of this difficulty, each transmission grating produced for MENA had somewhat different rejection/transmission characteristics. In addition, two different "lots" were manufactured for MENA: Lot U02 and Lot U04. In total, approximately 77 gratings were mounted in frames for possible use in MENA. To keep track of them all, an inventory numbering system was devised to identify the lot number, the wafer ID and the frame number.

After defects were repaired (patching pinholes) and the UV-rejection and ion transmission characteristics of each grating/frame assembly were measured, 15 were selected for the flight instrument-three from lot U02 and 12 from lot U04. Table II indicates which gratings went into each of the MENA heads.

A series of scanning electron micrographs (SEMs) of a MENA transmission grating are presented in Fig. 6. The frame ID for this grating assembly is IB030. It comes from
Lot U02 and has wafer and scribe ID numbers of U02H007 and 2994, respectively. Due to a variety of defects, this grating was not considered flight quality. The large rectangular hole in the grating was intentionally cut with an ion beam in order to measure the cross-sectional dimensions of the gold bars and fine nickel support structures.

\section{Fine nickel supports}

The fine nickel bars that support the gold gratings have either a rectangular or a slightly trapezoidal cross section as shown schematically in Fig. 7. The angle $\beta$ is measured from the normal incidence direction perpendicular to the collimating bars while the angle $\alpha$ is measured from the normal incidence direction parallel to the collimating bars. Given the symmetry of the collimating structures, we note that $\tau(+\alpha, \beta)=\tau(-\alpha, \beta)$ and $\tau(\alpha,+\beta)=\tau(\alpha,-\beta)$.

For the rectangular geometry, the probability of getting through the structure can be computed as shown in Fig. 7(b). From this figure, we can see that if a beam comprised of 

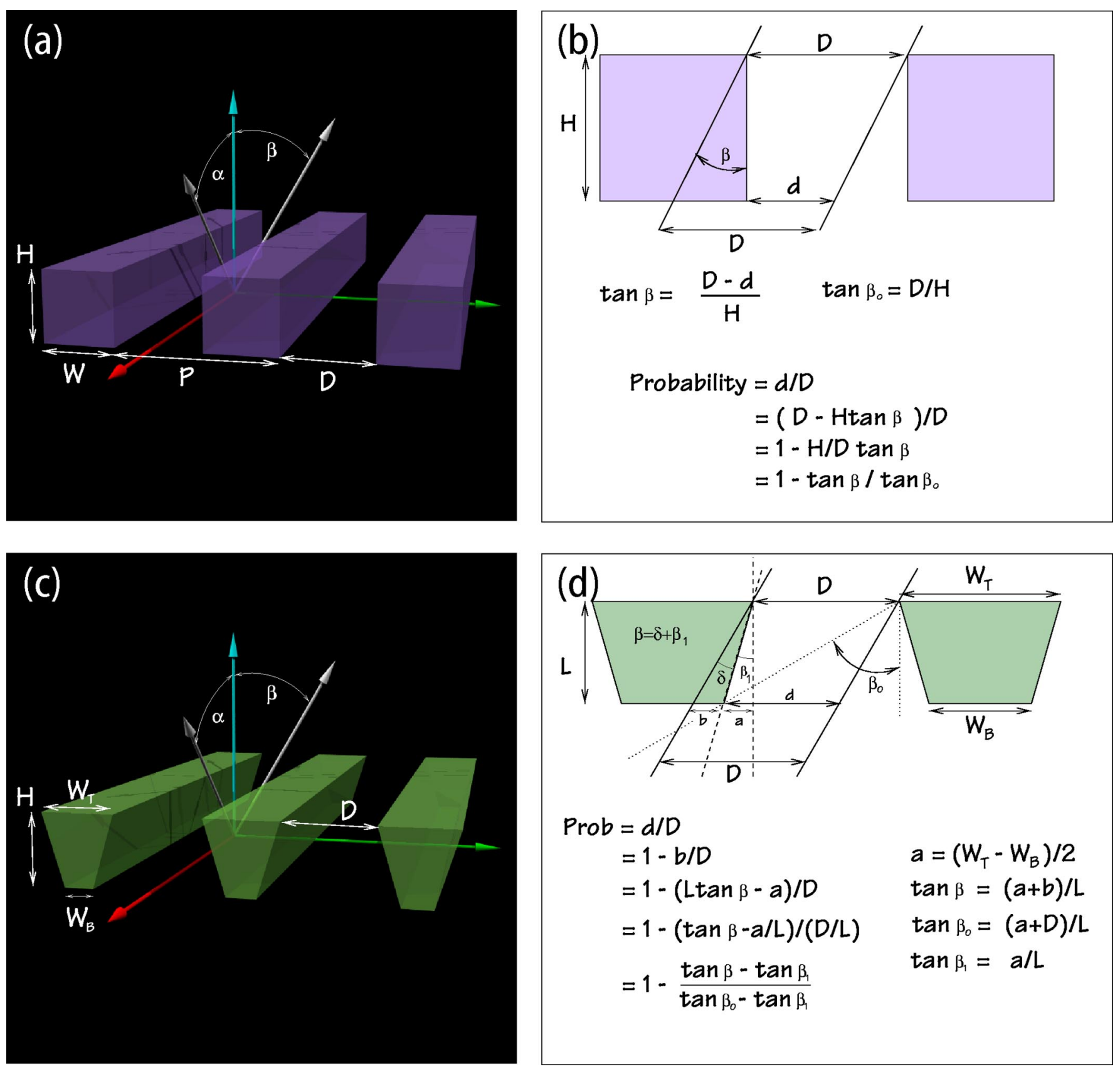

FIG. 7. (Color online). Schematic illustration of collimating structures with (a) rectangular and (b) trapezoidal cross sections. The transmission probabilities are given in (b) and (d).

parallel rays (e.g., from a distant source) impinges on the gap between collimating elements at an angle $\beta$, then only a fraction $d / D$ makes it through. Since $d=D-H \tan \beta$ and $\tan \beta$ 。 $=D / H$ (see figure), where $\beta_{\circ}$ is the maximum angle that can make it through, we have for the transmission probability, $\tau=1-\tan \beta / \tan \beta_{\text {。 }}$.

Note that in terms of the angles $\alpha$ and $\beta$-which are the angles in the parallel and perpendicular planes, respectively - the transmission probability is independent of $\alpha$.

An additional factor for the overall transmission probability needs to be taken into account: the probability of actually hitting a gap as opposed to hitting a bar. This probability is just given by $\varepsilon=D /(D+W)$, where $D$ is the gap width and $W$ is the bar (or plate) width. Thus, the overall transmission probability for a collimating structure with rectangular bars is given by

$$
\tau(\alpha, \beta)=\varepsilon\left\{\begin{array}{ll}
1-\frac{\tan \beta}{\tan \beta_{\circ}} & |\beta|<\beta_{\circ} \\
0 & \text { else }
\end{array},\right.
$$

$\tan \beta_{\circ}=\frac{D}{H}$,

$$
\varepsilon=\frac{D}{(D+W)}
$$


For the trapezoidal geometry, the probability of getting through the structure can be computed as shown in Fig. 7(d). Proceeding as above, the overall transmission probability for this collimating structure is given by

$$
\begin{aligned}
& \tau(\alpha, \beta)=\varepsilon \begin{cases}1 & |\beta|<\beta_{1}(23) \\
1-\frac{\tan \beta-\tan \beta_{1}}{\tan \beta_{\circ}-\tan \beta_{1}} & \beta_{1}<|\beta|<\beta_{\circ} \\
0 & \text { else }\end{cases} \\
& \tan \beta_{\circ}=\frac{a+D}{H}, \\
& \tan \beta_{1}=\frac{a}{H}, \\
& \varepsilon=\frac{D}{\left(D+W_{T}\right)}, \\
& a=\frac{W_{T}-W_{B}}{2},
\end{aligned}
$$

which is again independent of $\alpha$.

The actual dimensions of the fine nickel support structures were determined from measurements of SEMs as shown in Fig. 6(c). Although there is probably a slight trapezoidal shape to the fine nickel support bars, the SEMs analyzed so far suggest that the departure from a rectangular geometry is very small. Thus, assuming the nickel bars are rectangular in cross section, the dimensions obtained from Fig. 6(d) indicate that average bar width, height and period are $1.13,0.93$, and $3.96 \mu \mathrm{m}$, respectively.

\section{Curved collimator plates}

The large curved collimator plates on MENA (i.e., the brass-colored plates shown at the top of Fig. 5) can be thought of as very narrow and tall rectangular bars that are curved into arc shapes. The above transmission probability for the rectangular geometry is still valid for this case if we note that the height of each bar decreases by a factor of $\cos \alpha$. Thus, for the curved collimators, the transmission probability is dependent on $\alpha$ because the value of $\beta_{\circ}$ increases as $\alpha$ increases.

\section{Gold grating bars}

Although the gold grating bars were intended to have a rectangular geometry, technical limitations in their fabrication lead to bars that have a more "barrel-shaped" geometry. By this we mean that the cross section of each bar is like a rectangle with "bulging" sides (i.e., each bar looks like a barrel when viewed edge on). Here we develop an analytic expression for the transmission function associated with such structures under the assumption that the bulging sides can be expressed as arcs of circles.

The barrel geometry can be approximated by the intersection of a rectangular bar and two cylinders as shown in Figs. 8(a) and 8(b). From the lower part of panel (a), we can see what the radius of each circle needs to be, given that we know the height $h$ of a bar and the distance $c$ that the middle of the bar sticks out relative to the tops and bottoms of the bar

$$
r=\frac{h^{2}+4 c^{2}}{8 c} .
$$

If the gap between two bars is given by $D$, and we place the origin at the center of the gap, then the circle defining the left side of the gap is centered at a point $-(D / 2+r)$, while the circle defining the right side of the gap is centered at a point $+(D / 2+r)$.

As shown in Fig. 8(c), the transmission probability, $\tau$, is given by the quantity $d / D$, where $D$ is the (known) gap width and the distance $d$ is a function of the angle $\beta$. Note that, as with the rectangular and trapezoidal geometries discussed above, since $\beta$ is defined as the angle between the $y$ axis and the projection of the ray onto the $x-y$ plane, the transmission probability will be independent of the angle $\alpha$ (which is defined as the angle between the $y$ axis and the projection of the ray onto the $y-z$ plane).

In order to compute the transmission probability for this type of collimating structure, we note that there are three distinct cases to consider. The first case occurs when the incident rays enter the gap such that they are tangent to the cylindrical portions of the barrels. This will occur when $|\beta|$ $<\beta_{0}$. The second case occurs when $\beta_{0}<|\beta|<\beta_{1}$. In this case the incident rays are tangent to the cylinders at points that are not on the defined barrel. In this regime, $d$ is calculated simply by considering the corner points as we did for the rectangular geometry. Finally, the third case occurs when $|\beta|>\beta_{1}$. For this case no rays can get through and the probability must be 0 .

From Fig. 8(d), we can see that the limiting angles are given by

$$
\begin{aligned}
& \sin \beta_{0}=h / 2 r, \\
& \tan \beta_{1}=(2 c+D) / h .
\end{aligned}
$$

Also, from Fig. 8(b), we see that for $|\beta|<\beta_{0}$

$$
d=D+2 r\left(1-\frac{1}{\cos \beta}\right)
$$

and, since $\tau=d / D$,

$$
\tau=1+\frac{h^{2}+4 c^{2}}{4 c D}\left(1-\frac{1}{\cos \beta}\right) .
$$

When $\beta_{0}<|\beta|<\beta_{1}$, the transmission probability can be computed as we did for the simple rectangular bars with a small modification. Note that $D$ in this case is not the distance between the corner points-it is the gap between the bulging centers. So in this case, $d+h \tan \beta=D+2 c$, so that 

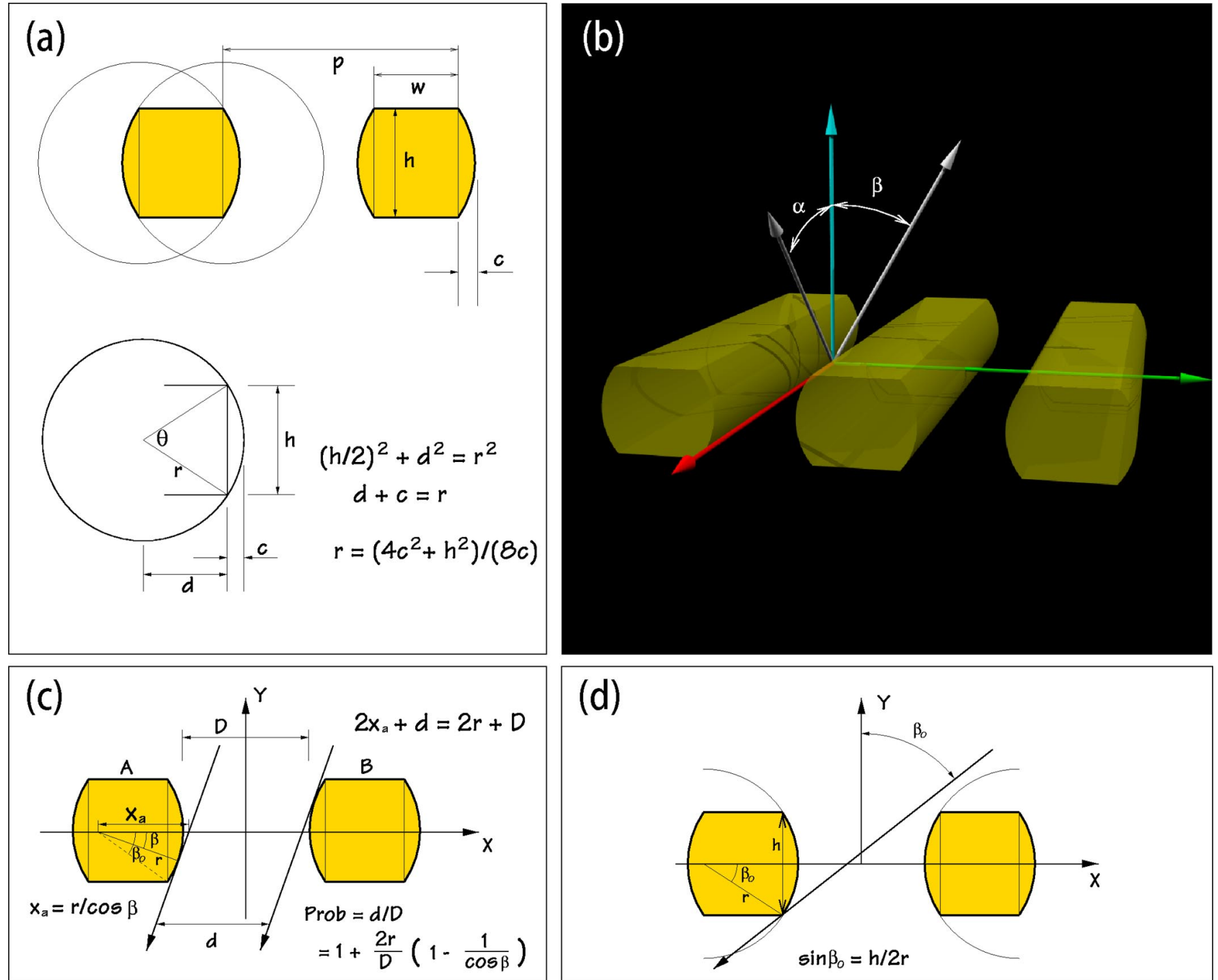

(d)

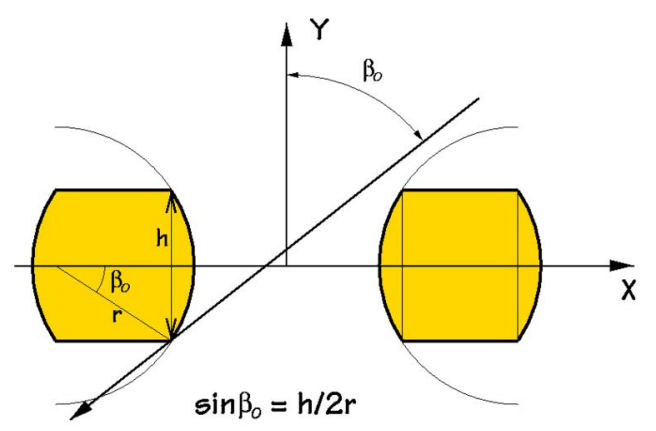

FIG. 8. (Color online) (a, b) Defining the "barrel" geometry in terms of intersecting cylinders. (c) The transmission probability that results when the incident parallel rays are tangent to the cylindrical sidewalls. (d) When $\beta>\beta_{o}$, the rays are no longer tangent to the cylindrical sidewalls. In this regime, the problem reduces to that of the rectangular cross section shown in Fig. 7.

$$
\tau=d / D=1+\frac{2 c}{D}-\frac{h}{D} \tan \beta
$$

Combining all three cases together we finally have the transmission probability for the gold bars

$$
\begin{aligned}
& \tau(\beta)=\varepsilon \begin{cases}\tau_{1}, & |\beta|<\beta_{0} \\
\tau_{2}, & \beta_{0}<|\beta|<\beta_{1} \\
0, & |\beta|>\beta_{1}\end{cases} \\
& \tau_{1}=1+\frac{h^{2}+4 c^{2}}{4 c D}\left(1-\frac{1}{\cos \beta}\right) \\
& \tau_{2}=1+\frac{2 c}{D}-\frac{h}{D} \tan \beta \\
& \sin \beta_{0}=h / 2 r \\
& \tan \beta_{1}=\frac{(D+2 c)}{h}
\end{aligned}
$$

$$
\varepsilon=\frac{D}{D+w} .
$$

The dimensions of the gold bars present in grating IB030 (from lot U02) were measured from the SEM shown in Fig. 6(d). The average height, width, period and gap size were determined to be $308,169,205$, and $36 \mathrm{~nm}$, respectively.

\section{Coarse nickel supports}

The coarse triangular nickel support mesh is a much more macroscopic structure than either the fine nickel support bars or the gold grating bars. In addition, as shown in the scanning electron micrograph in Fig. 9(a), it lies in the same layer and has the same height as the fine nickel support bars. As a result, the modification of the overall angular dependence of the transmission characteristics due to the coarse mesh will be very small. Thus, for the coarse triangular mesh we assume a uniform constant transmission factor of 0.899 . This value is derived from the dimensions shown in Fig. 9(b). 


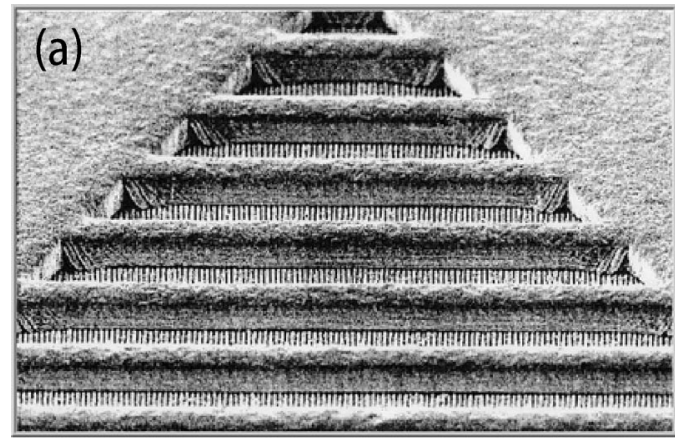

(b)

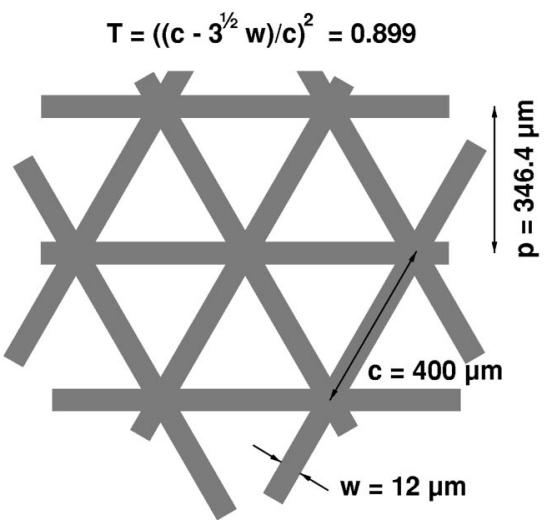

FIG. 9. (a) SEM showing a corner region of the coarse nickel mesh. The fine nickel bars are the medium-sized horizontal structures and the much smaller gold grating bars can be seen as vertical white stripes below them. Note that the coarse mesh is the same height as the fine nickel supports and therefore does not significantly alter the transmission characteristics. (b) Dimensions and transmission efficiency, $T$, of the coarse triangular nickel support mesh (not to scale).

\section{B. Overall transmission efficiency}

\section{Collimating structures}

The transmission functions for the curved collimator plates, the gold bars, and the fine nickel support bars in terms of the coordinate system defined in Fig. 2(a) are given by

$$
\begin{aligned}
& \tau_{C}(\xi, \phi)=\varepsilon_{C} \begin{cases}1-\frac{\tan \phi}{\tan \phi_{\circ, C}} & |\phi|<\phi_{\circ, C} \\
0 & \text { else }\end{cases} \\
& \tau_{\mathrm{TG}}(\phi)=\varepsilon_{\mathrm{TG}} \begin{cases}\tau_{1}, & |\phi|<\phi_{0, \mathrm{TG}} \\
\tau_{2}, & \phi_{0, \mathrm{TG}}<|\phi|<\phi_{1, \mathrm{TG}} \\
0, & |\phi|>\phi_{1, \mathrm{TG}}\end{cases} \\
& \tau_{\mathrm{NS}}(\xi)=\varepsilon_{\mathrm{NS}} \begin{cases}1 & |\xi|<\xi_{1} \\
1-\frac{\tan \xi-\tan \xi_{1}}{\tan \xi_{\circ}-\tan \xi_{1}} & \xi_{1}<|\xi|<\xi_{\circ} \\
0 & \text { else }\end{cases} \\
& \tau_{\mathrm{CM}}=0.899 \\
& \tau_{1}=1+\frac{h^{2}+4 c^{2}}{4 c D}\left(1-\frac{1}{\cos \phi}\right) \\
& \tau_{2}=1+\frac{2 c}{D}-\frac{h}{D} \tan \phi
\end{aligned}
$$

$$
\begin{aligned}
& \tan \phi_{\circ, C}=\frac{D_{C}}{H_{C} \cos \xi} \\
& \sin \phi_{0, \mathrm{TG}}=H_{\mathrm{TG}} / 2 r \\
& \tan \phi_{1, \mathrm{TG}}=\frac{\left(D_{\mathrm{TG}}+2 c\right)}{H_{\mathrm{TG}}} \\
& \tan \xi_{\circ}=\frac{\left(a_{\mathrm{NS}}+D_{\mathrm{NS}}\right)}{H_{\mathrm{NS}}} \\
& \tan \xi_{1}=\frac{a_{\mathrm{NS}}}{H_{\mathrm{NS}}} \\
& a_{\mathrm{NS}}=\frac{W_{T}-W_{B}}{2} \\
& \varepsilon_{C}=\left(\frac{D_{C}}{D_{C}+W_{C}}\right) \\
& \varepsilon_{\mathrm{TG}}=\left(\frac{D_{\mathrm{TG}}}{D_{\mathrm{TG}}+W_{\mathrm{TG}}}\right) \\
& \varepsilon_{\mathrm{NS}}=\left(\frac{D_{\mathrm{NS}}}{D_{\mathrm{NS}}+W_{T, \mathrm{NS}}}\right) .
\end{aligned}
$$

The subscripts C, TG, NS, and CM refer to the Collimator, Transmission Grating, Nickel Support, and Coarse Mesh structures, respectively. Note that we have left these terms as functions of $\xi$ and $\phi$ for clarity. It is a simple task to make them functions of $\theta$ and $\phi$ instead.

\section{Energy dependence}

Since all atoms with energies above about $1 \mathrm{keV}$ are able to make it through the foil, the transmission efficiency above $1 \mathrm{keV}$ is taken to be $100 \%$. Below $1 \mathrm{keV}$ the transmission efficiency will begin to drop due to the finite thickness of the carbon foil. In this regime, the efficiency will also be dependent upon the angle of incidence since the path length through the foil is dependent upon the angle of incidence. A detailed calculation of these efficiencies can be made using the TRIM code, ${ }^{5}$ but for our present purposes we will simply restrict our analysis to energies above $1 \mathrm{keV}$ and assume (to a good approximation) that there is no energy dependence to the transmission efficiency.

\section{Postfoil detection efficiency}

For ENAs that make it through the collimator, transmission grating and foil, an additional postfoil detection efficiency must be taken into account. This additional efficiency results from a number of individual efficiencies including: the probability of generating secondary electrons in the foil; the probability of detecting the primary ENA; the probability of detecting the secondary start electrons; and the probability of making it through the accelerating and shields grids. Although this combined efficiency is not easy to compute from first principles, it can be measured in a relatively simple 
TABLE III. MENA collimator dimensions and transmission efficiencies.

\begin{tabular}{ccccc}
\hline \hline $\begin{array}{c}\text { Parameter } \\
\text { (meaning } \\
\text { varies) }\end{array}$ & $\begin{array}{c}\text { Curved } \\
\text { collimator } \\
\text { plates }\end{array}$ & $\begin{array}{c}\text { Gold } \\
\text { transmission } \\
\text { grating bars }\end{array}$ & $\begin{array}{c}\text { Fine } \\
\text { nickel } \\
\text { support bars }\end{array}$ & $\begin{array}{c}\text { Coarse } \\
\text { nickel } \\
\text { mesh }\end{array}$ \\
\hline $\mathrm{P}$ & $0.4671 \mathrm{~cm}$ & $205 \mathrm{~nm}$ & $3.96 \mu \mathrm{m}$ & $346.4 \mu \mathrm{m}$ \\
$\mathrm{D}$ & $0.4417 \mathrm{~cm}$ & $16.27 \mathrm{~nm}$ & $2.83 \mu \mathrm{m}$ & $\ldots$ \\
$\mathrm{H}$ & $6.3144 \mathrm{~cm}$ & $308 \mathrm{~nm}$ & $0.93 \mu \mathrm{m}$ & $0.93 \mu \mathrm{m}$ \\
$\mathrm{W}$ & $0.0254 \mathrm{~cm}$ & $169 \mathrm{~nm}$ & $1.13 \mu \mathrm{m}$ & $12 \mu \mathrm{m}$ \\
$\mathrm{C}$ & $\ldots$ & $9.87 \mathrm{~nm}$ & $\ldots$ & $400 \mu \mathrm{m}$ \\
$\mathrm{T}_{\mathrm{f}}$ & $94.6 \%$ & $7.93 \%$ & $71.5 \%$ & $89.9 \%$ \\
\hline \hline
\end{tabular}

manner in the laboratory using a combination of the coincidence and singles rates from the start and stop portions of the detector.

If ENAs are passing through the foil at a rate $S$ and at an angle of incidence such that the parallel-projected area of the entrance aperture falls completely within the active area of the stop detector, then the individual singles rates in the start and stop detectors will be given by: $R_{A}=\varepsilon_{A} S$ and $R_{B}=\varepsilon_{B} S$, respectively. Furthermore, if the detectors are independent, the coincidence rate will be: $R_{A B}=\varepsilon_{A} \varepsilon_{B} S+R_{\mathrm{ch}}=\varepsilon S+R_{\mathrm{ch}}$ where the overall postfoil detection efficiency, $\varepsilon$, is equal to $\varepsilon_{A} \varepsilon_{B}$. Here, $R_{\mathrm{ch}}$ is the chance coincidence rate which is approximately given by $T R_{A} R_{B}$, where $T$ is the coincidence time window. In our case, the overall postfoil efficiency is near $40 \%$ and the laboratory measurements were taken with singles rates on the order of $1 \mathrm{kHz}$ and a time window of approximately $300 \mathrm{~ns}$. Therefore (in the laboratory), $R_{\mathrm{ch}}$ is negligible in comparison to the total measured coincidence rate, $R_{A B}$, and can be safely neglected.

Since $S=R_{A B} / \varepsilon$, we have $\varepsilon_{A} \varepsilon_{B}=\left(\varepsilon R_{A} / R_{A B}\right)$ $\times\left(\varepsilon R_{B} / R_{A B}\right)=\varepsilon$. Thus

$$
\varepsilon=\frac{\left(R_{A B}\right)^{2}}{R_{A} R_{B}} .
$$

Use of this formula together with measurements of the coincidence and singles rates taken in the laboratory prior to launch gives a total overall postfoil detection efficiency of $\varepsilon=0.424$.

\section{Overall detection efficiency}

Finally, a total transmission probability as a function of look direction can be defined as the product of all the relevant probabilities

$$
\tau(\theta, \phi)=\tau_{C}(\phi) \times \tau_{\mathrm{TG}}(\theta, \phi) \times \tau_{\mathrm{NS}}(\theta, \phi) \tau_{\mathrm{CM}} \times \varepsilon .
$$

The various dimensions assumed for the three collimating structures are summarized in Table III and plots showing the dependence of $\tau_{C}, \tau_{\mathrm{TG}}, \tau_{\mathrm{NS}}$, and $\tau$ on the angles $\alpha$ and $\phi$ are shown in Fig. 10.

\section{GEOMETRIC FACTORS}

For each start and stop-byte combination, we can define a geometric factor as follows:

$$
G_{i j k}=\int_{\theta=-\pi / 2}^{+\pi / 2} \int_{\phi=0}^{\pi} A_{i j k}(\theta, \phi) \tau(\theta, \phi) \cos \theta d \theta d \phi .
$$

Here, $i$ and $j$ correspond to start- and stop-byte numbers and $k$ corresponds to the head number $(0,1$, or 2$)$.

The $G_{i j k}$ for IMAGE/MENA were computed (numerically) and are shown in Fig. 11. Note that there is a very significant degree of asymmetry in each head and that the three heads are all different. The anomalously low amplitudes for start bytes 4 and 14 are due to the fact that those start bytes correspond to physical entrance apertures at the edges of the grating and are therefore chopped to smaller sizes than those start bytes that map to the central regions of the grating. Similarly, start bytes $[0-3,15]$ all map to regions outside of the entrance aperture and therefore they all have $G \equiv 0$.

\section{CULLING OF DIRECT EVENTS}

For the sake of simplicity, we have so far ignored the fact that each head actually has a central blind spot. This blind spot exists because the section of the detector area below the start foil must be dedicated to detecting start electrons and cannot simultaneously detect ENAs. Therefore, in reality, the geometric factors for each start byte will drop very rapidly to zero for stop-byte values that map to the start section of the position sensitive detector. The exact stop-byte ranges where the count rates first begin to drop for each start byte can be easily determined from the data itself. We have compiled these values into a "mask" array which we use to "cull out" start/stop-byte pairs that map within or close to the edges of the central blind spot. This simple approach allows us to use the geometric factors shown in Fig. 11 without modification since the nonphysical regions will never be used. [Note that the specific start/stop-byte pairs that we cull can be seen as black areas in the angle look up tables (LUTs) shown later in Fig. 12(a).]

\section{COMPARISON WITH ABSOLUTE CALIBRATION}

The effective area at various incident angles for heads 1 and 2 in the MENA instrument were measured in the laboratory prior to launch. A broad beam source of $\mathrm{H}^{+}$and $\mathrm{O}^{+}$at $31 \mathrm{keV}$ and of $\mathrm{H}^{+}$at $9 \mathrm{keV}$ were used for this purpose. The beam was broad enough to fill the entrance aperture and was measured to have very low divergence $\left(\approx 0.1^{\circ}\right)$ so that, to a very good approximation, it can be considered as a parallel- 

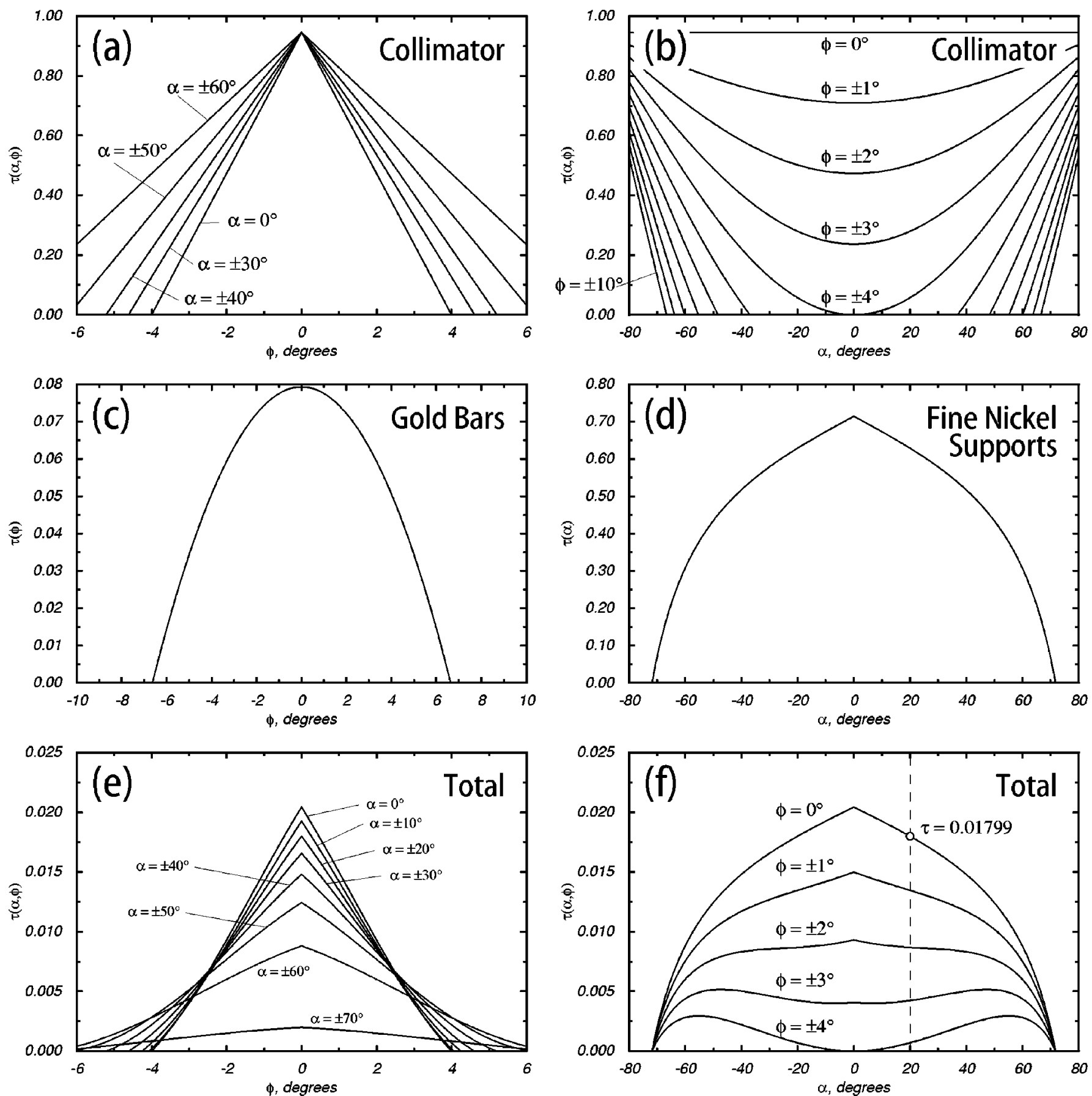

FIG. 10. Angular dependence of the transmission probabilities for the various collimating structures in MENA. (a) As a function of $\phi$ for the curved collimator plates. Note that $\tau$ is dependent on both $\alpha$ and $\phi$ for the collimator plates. (b) As a function of $\alpha$ for the curved collimator plates. (c) As a function of $\phi$ for the gold transmission grating bars assuming they have barrel-shaped cross sections. Note that $\tau$ is independent of $\alpha$ in this case. (d) As a function of $\alpha$ for the fine nickel supports. Note that $\tau$ is independent of $\phi$ in this case. (e) Total transmission probability as a function of $\phi$. Curves for $\alpha=0^{\circ}, \pm 10^{\circ}, \pm 20^{\circ}, \pm 30^{\circ}$, $\pm 40^{\circ}, \pm 50^{\circ}, \pm 60^{\circ}, \pm 70^{\circ}$, and $\pm 80^{\circ}$ are shown. (f) Total transmission probability as a function of $\alpha$. Curves for $\phi=0^{\circ}, \pm 1^{\circ}, \pm 2^{\circ}, \pm 3^{\circ}$, and $\pm 4^{\circ}$ are shown.

ray source. The beam was also imaged with a twodimensional (2D) imager in order to verify that it was spatially uniform to a high degree. The omni-directional flux $J$ of the beam (in units of number $/ \mathrm{cm}^{2} / \mathrm{s}$ ) was measured with a Faraday cup detector. The beam was then directed into the MENA instrument heads at various angles of incidence in order to determine the total coincidence (or "valids") rate, $C$. The effective area of a head at a given angle of incidence can then be calculated as $A_{\text {eff }}=C / J$. As discussed elsewhere, the results for head 3 are unreliable. This is because the $1 \mathrm{keV}$ foil bias voltage cable separated from the instrument during calibration runs. However, the data for heads 1 and 2 give effective areas as follows:

$$
\begin{aligned}
& A_{1, \mathrm{eff}}=0.10 \pm 0.04 \mathrm{~cm}^{2}, \\
& A_{2, \mathrm{eff}}=0.11 \pm 0.06 \mathrm{~cm}^{2} .
\end{aligned}
$$

To relate these measurements to our calculations given above, we note that if we are only interested in the total 

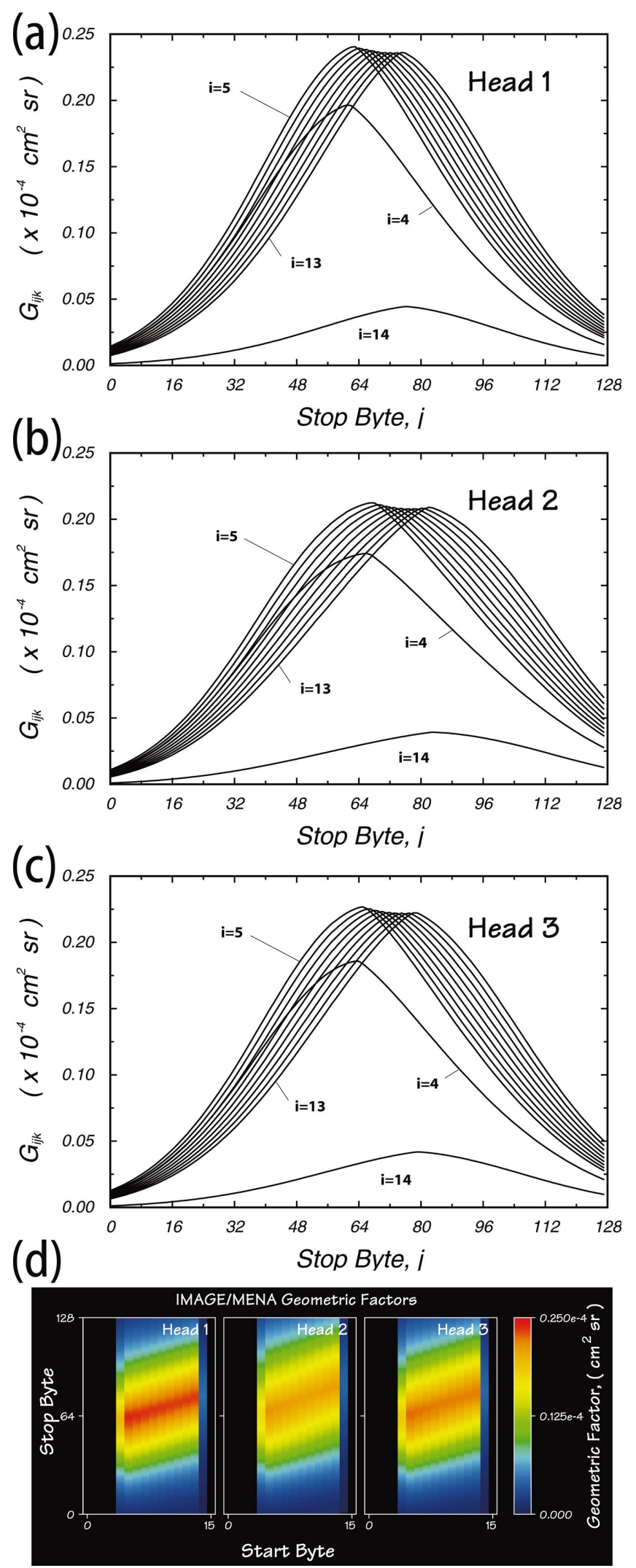

FIG. 11. (Color) Geometric factors for heads 1, 2, and 3. Each curve represents a different start-byte value (4-14). The geometric factors for start-byte values of 0-3 and 15 are all zero because those byte values do not map to physical locations in the entrance aperture. Similarly, start-byte values of 4 and 14 give lower G, because they map to regions near the edge of the entrance aperture and are somewhat truncated as a result.

coincidence (or valids) rate, for a given start byte, and a given incident angle, we can consider the projected area as the sum of all the projected areas obtained from each stop byte. Note that only a few stop bytes will contribute anything to the sum for a given start byte and incident angle. For head 2 , the total projected area at $\theta=20^{\circ}$ is shown in Table IV 
' 0

File Controls Integral Differential Testing Help

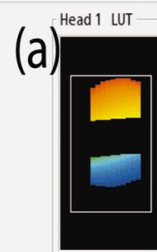

Head 1 Count Rate v Stop Byte

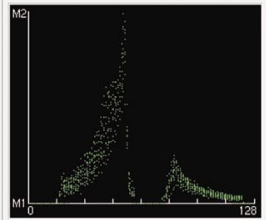

Head 2 LUT -

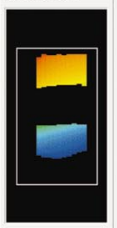

Head 3 LUT -

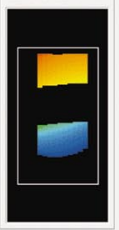

20001005

Date: 2000279

UT: 14:00:00

Spins: 60
dT: $120 \mathrm{~min}$

Energy: $1.0-1000.0 \mathrm{keV}$

M1: 0.00

M2: 1273.67

J1: $10^{\wedge} 1.00$

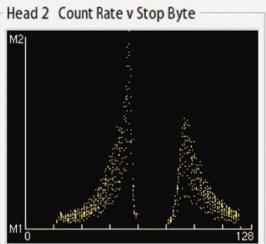

Head 3 Count Rate v Stop Byte

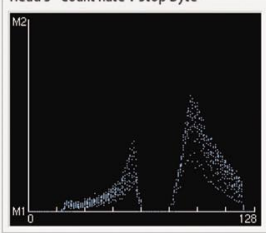

Combined Count Rate v Stop Byte

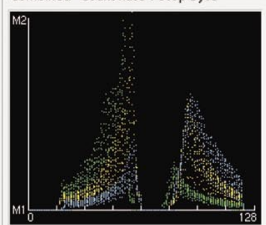

IMAGE/MENA XMEna

$\theta \theta \theta$

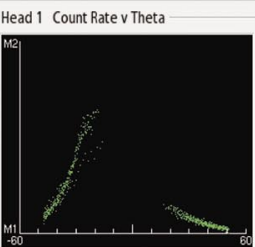

Head 2 Count Rate v Theta

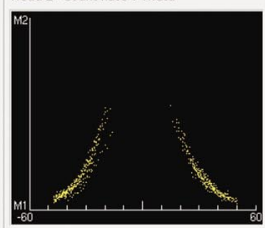

Head 3 Count Rate $v$ Theta

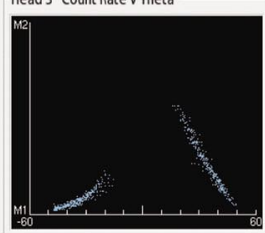

Combined Count Rate v Theta

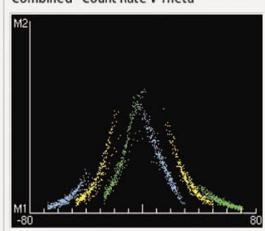

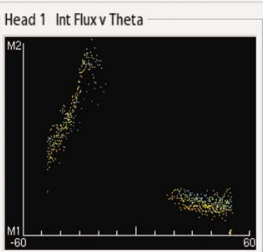

Head 2 Int Fluxv Theta

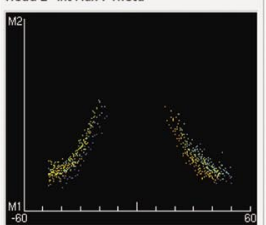

Head 3 Int Fluxv Theta

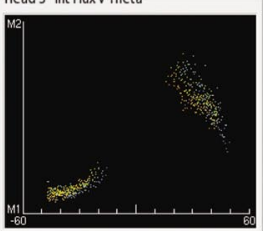

Combined Int Fluxv Theta -

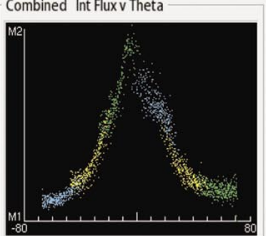

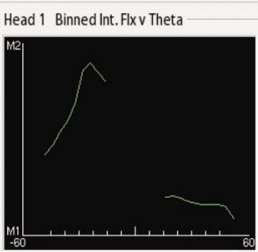

Head 2 Binned Int. Flxv Theta

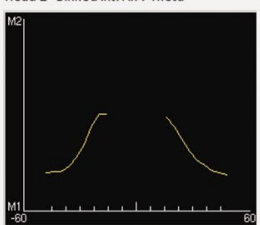

Head 3 Binned Int. Flxv Theta

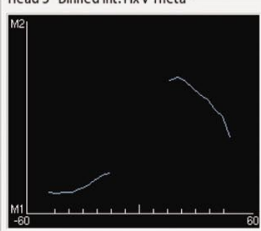

Combined Binned Int. Flxv Theta

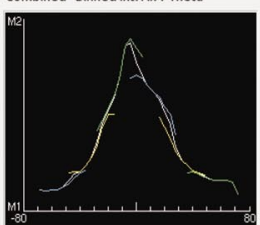

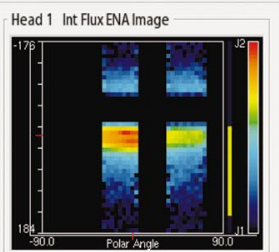

Head 2 Int Flux ENAImage

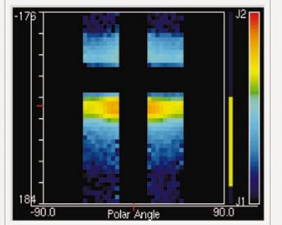

Head 3 Int Flux ENAImage

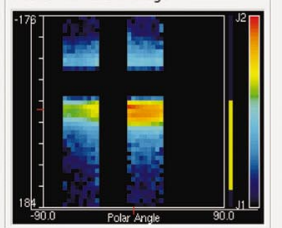

Combined Int Flux ENA Image

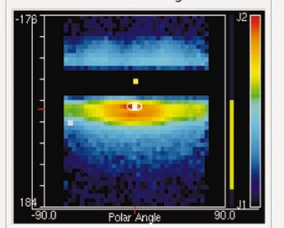

(b)

\section{IMAGE/MENA Energetic Neutral Atom Imagery} October 5, 2000 (2000279)

1400:00 UT $(1300: 00-1500: 00)$

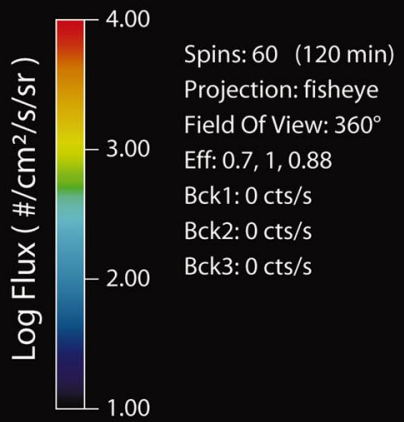

Energy: > 1 keV (1-1000 keV)

Pixel Size (Polar x Azimuth): $4^{\circ} \times 8^{\circ}$

IMAGE Ephemeris (GSM):

Position: -5.27, 0.57, 5.69 Re

Spin Axis: $-0.03,0.99,-0.13$

\section{- LosAlamos}

FIG. 12. (Color) (a) Main window of the IMAGE/MENA interactive data processing tool. Data from a storm interval on October 5, 2000 is shown. The ENA images are (effectively) integral flux images for $\mathrm{E} \geqslant 1 \mathrm{keV}$. The broad cyan colored regions are due to ENAs emitted from the plasma sheet. (b) Projected version of the image shown in (a). 
TABLE IV. Calculated effective areas at $\theta=20^{\circ}$ for head 2 .

\begin{tabular}{|c|c|c|c|c|}
\hline Start byte & Stop bytes & $\begin{array}{l}\text { Projected area } \\
\mathrm{cm}^{2}\end{array}$ & $\tau$ & $\begin{array}{l}\text { Effective area } \\
\mathrm{cm}^{2}\end{array}$ \\
\hline 0 & $\cdots$ & 0.00000 & 0.01799 & 0.00000 \\
\hline 1 & $\cdots$ & 0.00000 & 0.01799 & 0.00000 \\
\hline 2 & $\ldots$ & 0.00000 & 0.01799 & 0.00000 \\
\hline 3 & $\cdots$ & 0.00000 & 0.01799 & 0.00000 \\
\hline 4 & $49-50$ & 0.61080 & 0.01799 & 0.01099 \\
\hline 5 & $50-51$ & 0.75175 & 0.01799 & 0.01353 \\
\hline 6 & $51-53$ & 0.75175 & 0.01799 & 0.01353 \\
\hline 7 & $53-54$ & 0.75175 & 0.01799 & 0.01353 \\
\hline 8 & $54-56$ & 0.75175 & 0.01799 & 0.01353 \\
\hline 9 & $56-57$ & 0.75175 & 0.01799 & 0.01353 \\
\hline 10 & $57-59$ & 0.75175 & 0.01799 & 0.01353 \\
\hline 11 & $59-61$ & 0.75175 & 0.01799 & 0.01353 \\
\hline 12 & $61-62$ & 0.75175 & 0.01799 & 0.01353 \\
\hline 13 & $62-64$ & 0.75175 & 0.01799 & 0.01353 \\
\hline 14 & $64-64$ & 0.14095 & 0.01799 & 0.00254 \\
\hline 15 & $\ldots$ & 0.00000 & 0.01799 & 0.00000 \\
\hline & & \multicolumn{3}{|c|}{ Total effective area: $0.1353 \mathrm{~cm}^{2}$} \\
\hline
\end{tabular}

along with the overall transmission efficiency at $\theta=20^{\circ}$ and the resulting effective area. The "stop-bytes" column indicates which stop bytes actually contributed to the sum (for $\theta=20^{\circ}$ ). Summing the effective areas for each start byte gives a total effective area at $\theta=20^{\circ}$ of

$$
A_{2, \text { eff }}=0.1353 \mathrm{~cm}^{2} \text {. }
$$

Note from Table IV that the total projected area is $7.5175 \mathrm{~cm}^{2}$ which is just the total area of the gratings $\left(5 \times 1.0 \mathrm{~cm} \times 1.6 \mathrm{~cm}=8.0 \mathrm{~cm}^{2}\right)$ multiplied by $\cos \left(20^{\circ}\right)$. We could have guessed this from the outset, but the above analysis provides an excellent check on our calculations of the byte-dependent projected area curves (like those shown in Fig. 4).

Although there is considerable uncertainty in the measurements for the effective area, our calculated value for head 2 is reasonably close to the measured value of $0.11 \pm 0.06 \mathrm{~cm}^{2}$ and is well within the uncertainty range.

\section{INTEGRAL FLUX IMAGES}

From Eq. (5), the count rate for a given start byte ( $i$ index), stop byte $(j$ index $)$, head ID $(k$ index $)$, and azimuth bin $(\ell$ index $)$ is given by

$$
C_{i j k \ell}=\int_{E} j_{0, i j k \ell}(E) \Gamma_{i j k}(E) d E .
$$

Since the angular distribution of the differential flux does not vary much over a start-byte/stop-byte pair, we can safely set $F(\theta, \phi)=1$ so that $\Gamma_{i j k}(E)=G_{i j k}(E)$. Furthermore, if the overall detection efficiency is independent of energy (for energies above about $1 \mathrm{keV}$ this is a good approximation), then $G_{i j k}(E)=G_{i j k}$. We then have

$$
C_{i j k \ell}=G_{i j k} \int_{E} j_{0, i j k \ell}(E) d E .
$$

To define an "integral flux," $J_{i j k l}$, we accumulate counts from a given lower cutoff energy, $E_{o}$, up to $E=\infty$. Then

$$
C_{i j k \ell}=G_{i j k} \int_{E_{\circ}}^{\infty} j_{0, i j k \ell}(E) d E=G_{i j k} J_{i j k \ell} .
$$

The integral flux, $J$, is therefore given by

$$
J_{i j k \ell}=\frac{C_{i j k \ell}}{G_{i j k}} .
$$

To perform this calculation for the IMAGE/MENA direct-events data, for each head and azimuth bin, counts are first accumulated into stop-byte/start-byte bins. Then the total count rate for that bin, $C_{i j k \ell}$, is divided by the corresponding geometric factor, $G_{i j k}$. Each of the resulting integral fluxes are then mapped to the polar angle implied by their $i j k$ values. The interactive graphical tool shown in Fig. 12(a) illustrates this process step by step. The top three rows show the process for heads 1, 2, and 3 separately, while the bottom row shows all three heads combined. From left to right the top three rows show: (1) the polar angle look up table (LUT) as a function of start (horizontal dimension) and stop (vertical dimension) bytes; (2) the raw total count rate as a function of stop byte for start bytes of $0-15$ (i.e., there are in essence 16 curves of count rate versus stop byte overplotted); (3) the raw total count rate as a function of polar angle (each bin's start and stop byte gives a polar angle via the angle LUTs); (4) integral flux (count rate divided by G) as a function of polar angle; (5) integral flux binned into $4^{\circ}$ polar angle bins; (6) complete all-sky image for a given head with azimuth angle on the vertical axis and the polar angle on the horizontal axis.

All of the line plots are averaged over the azimuthal bins indicated by the yellow bar that appears to the right of each all-sky image. For the top three rows, the polar angle plots range from $-60^{\circ}$ to $60^{\circ}$ and are all in the "head frame" (in which $\theta=0^{\circ}$ is perpendicular to the detector plane). In the bottom row, the individual heads are combined by shifting heads 1 and 3 by $\pm 20^{\circ}$ with respect to head 2 . In addition, the plots that are functions of polar angle range from $-80^{\circ}$ to $80^{\circ}$ in the bottom row. To reduce artifacts in the final merged image, note that we do not simply merge together the three individual head images. Instead, we bin the combined corrected angular distributions (i.e., the processing always flows from left to right in Fig. 12(a) — not top to bottom).

Although the geometric factors obtained do an excellent job of converting counts to flux, combining data from the three heads together almost always produces artifacts in the final image if additional processing is not performed. The two main reasons for this are: (1) the overall relative efficiencies of the three heads may vary to some degree, and (2) there may be different amounts of background noise in each image. In addition, the background noise must be subtracted from the count rate, not the final fluxes. In the presence of background noise and an overall efficiency factor, the flux is given by

$$
J=\varepsilon\left(\frac{C-B}{G}\right) .
$$

In order to seamlessly merge data from the the three separate heads together, we first need to determine the relative efficiencies and the background count rates. Since we 


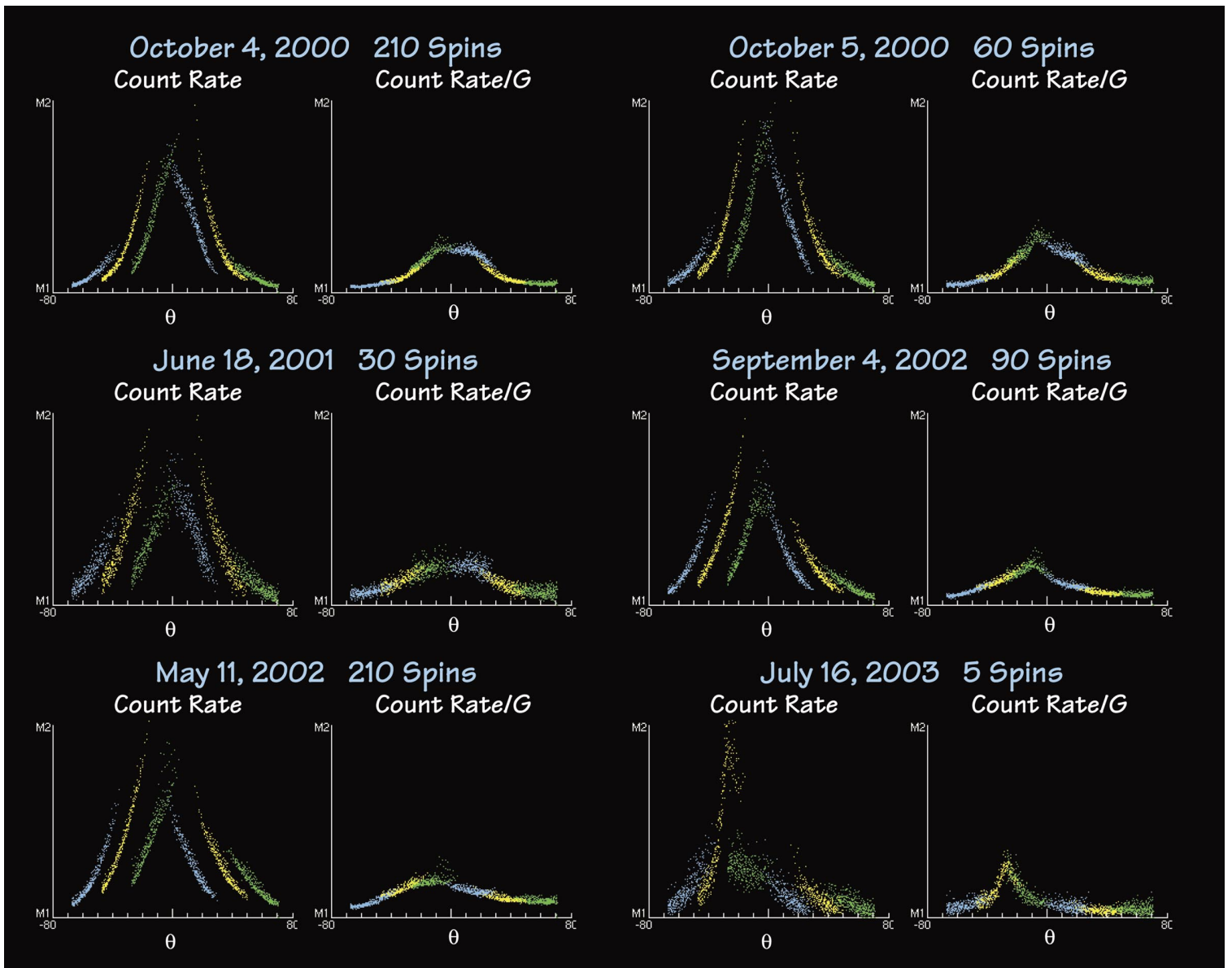

FIG. 13. (Color) Count rate vs polar angle and integral flux (count-rate/G) vs polar angle (for $E \geqslant 1 \mathrm{keV}$ ) for six different time periods. The contribution from each head is color coded (head 1 is green, head 2 is yellow, head 3 is blue). Many spins were used (except for the July 16 example) in order to reduce counting noise.

are only interested in relative efficiencies for this operation, we peg the overall efficiency of head 2 as $\varepsilon_{2} \equiv 1$ and let the efficiencies of heads 1 and $3\left(\varepsilon_{1}\right.$ and $\left.\varepsilon_{3}\right)$ as well as the background count rates in all three heads $\left(B_{1}, B_{2}\right.$, and $\left.B_{3}\right)$ vary as free parameters. We determine the five unknown parameters using a fitting procedure to minimize the total absolute difference (in log space) between the fluxes that result at polar angles where the three heads overlap. The problem is not under determined since there are five unknowns and five regions of overlap. More work needs to be done on which azimuth bins should be used for this fitting procedure, but the results so far are quite encouraging. In addition, strong emissions tend to dominate the fit which can sometimes produce obvious artifacts in very low flux regions. For this reason, we tentatively chose to fix the background rates at 0.0 in Fig. 12.

A projected version of the final integral flux image shown in Fig. 12(a) is presented in Fig. 12(b). The data are shown in a $360^{\circ}$ fish-eye projection looking toward the center of the earth. Gaps in coverage near the poles of the allsky image (polar angles approaching $\pm 90^{\circ}$ ) can be seen as distorted black circular regions to the left and right of the earth. The broad black horizontal gap connecting the poles corresponds to azimuth bins for which the instrument automatically turned off during its sweep past the sun. The bright regions above the sun gap are likely spurious counts due to solar UV photons. The grid seen through the data is the un- derlying equatorial plane of the solar-magnetic (SM) coordinate system and the lines are drawn every $2 R_{E}$ in both the $x$ and $y$ directions. The bright (yellow, orange and red) emissions are ENAs emitted from regions quite close to the earth, while the more extended dimmer emissions (blue to cyan) are emitted farther out from the plasma sheet. Note that in this somewhat peculiar projection, the inner part of the image (i.e., the circular region inside of the poles) corresponds to the more familiar $180^{\circ}$ fish-eye projection, while the ring outside of this corresponds to regions that are actually "behind us." While the mapping in the outer regions is not intuitively obvious (especially near the poles), this type of projection allows us to display/monitor all of the data available at once without adversely distorting the view in the earthward direction.

In Fig. 13, the count rate versus angle and the integral flux versus angle panels are shown enlarged for six different events. The contributions from each head are color coded: head 1 is green; head 2 is yellow; and head 3 is blue. As can be seen, in all cases the head-to-head match is excellent.

\section{DIFFERENTIAL FLUX IMAGES}

As in the previous section, the count rate for a given start byte ( $i$ index), stop byte ( $j$ index), head ID ( $k$ index), and azimuth bin $(\ell$ index $)$ is given by 


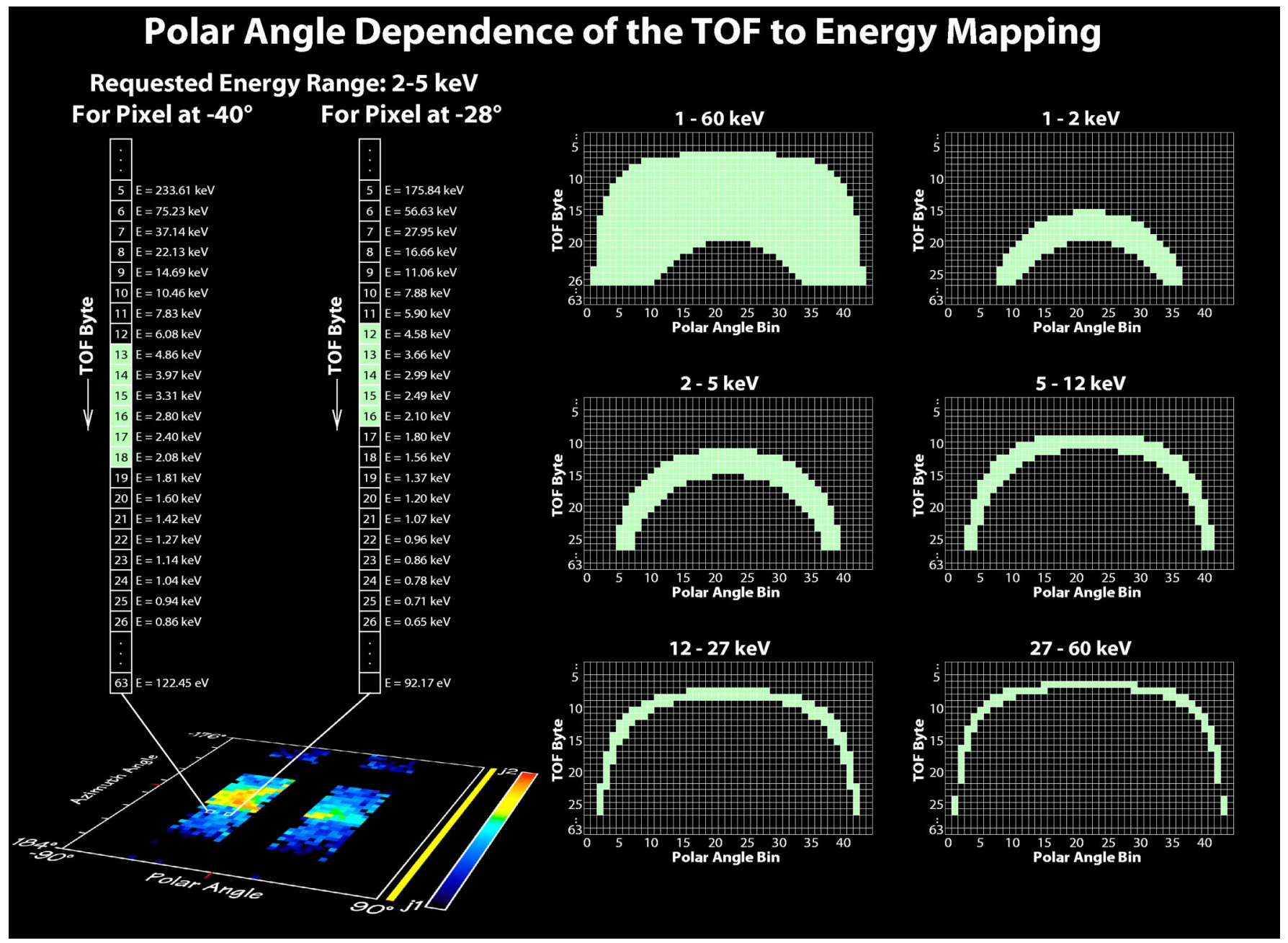

FIG. 14. (Color online) Time-of-flight (TOF) byte to energy mapping at two different polar angles. TOF bytes used for the requested energy bands as a function of polar angle bin. Note that the actual $E$ and $\Delta E$ implied by these groupings of bytes is what should be used in the flux conversion formula-not the requested $E$ and $\Delta E$.

$$
C_{i j k \ell}=\int_{E} j_{0, i j k \ell}(E) G_{i j k}(E) d E .
$$

If $j_{0, i j k \ell}(E)$ and $G_{i j k}(E)$ do not change much over the range of integration (e.g., spectrum is flat or $\Delta E$ is small), this expression can be approximated by

$$
C_{i j k \ell}=G_{i j k} j_{0, i j k \ell}(E) \Delta E .
$$

Thus

$$
j_{0, i j k \ell}(E)=\frac{C_{i j k \ell}}{G_{i j k}(E) \Delta E} .
$$

Technically, this equation is correct, but as we shall see in the next section its naive application leads to several problems.

\section{A. Problems with energy binning}

In order to determine the energy of a particle in the MENA instrument, we need to know: its time of flight (TOF); its angle of incidence; and its mass. If we assume for the moment that all of the ENAs are hydrogen, then we can ignore the mass dependence. Then, the angle of incidence gives the path length, the TOF and path length give the speed, and the speed and assumed mass give the energy. The main problem with this scheme arises as a result of two separate effects: (1) once measured, the TOF value is digitized into fairly coarse "TOF bytes" and (2) the mapping of TOF byte to energy changes as a function of polar angle (because the path length changes).

These effects are illustrated in Fig. 14. The image in the lower left portion of the figure is an integral flux image from head 2 and the two vertical arrays represent the 64 TOF byte values possible in the direct events data. The array on the left shows the TOF to energy mapping for a pixel at a polar angle of $-40^{\circ}$ while the array on the right shows the TOF to energy mapping for a pixel at a polar angle of $-28^{\circ}$. As can be seen, the midpoint energy for each TOF byte is somewhat different for the two pixels. The main effect of this variation is that for a given requested energy band, a different set of TOF bytes will end up getting used depending on what angle we are looking at. For example, if we ask for energies between 2 and $5 \mathrm{keV}$, the TOF bytes that will be used at $-40^{\circ}$ and $-28^{\circ}$ are those that are shaded green in Fig. 14. The right-hand side of Fig. 14 shows the TOF bytes that would be used for other requested energy bands at the midpoint of the nominal $4^{\circ}$ polar angle bins (note that we are not restricted to using $4^{\circ}$ bins).

At this point, it is important to recognize that the $\Delta E$ that 

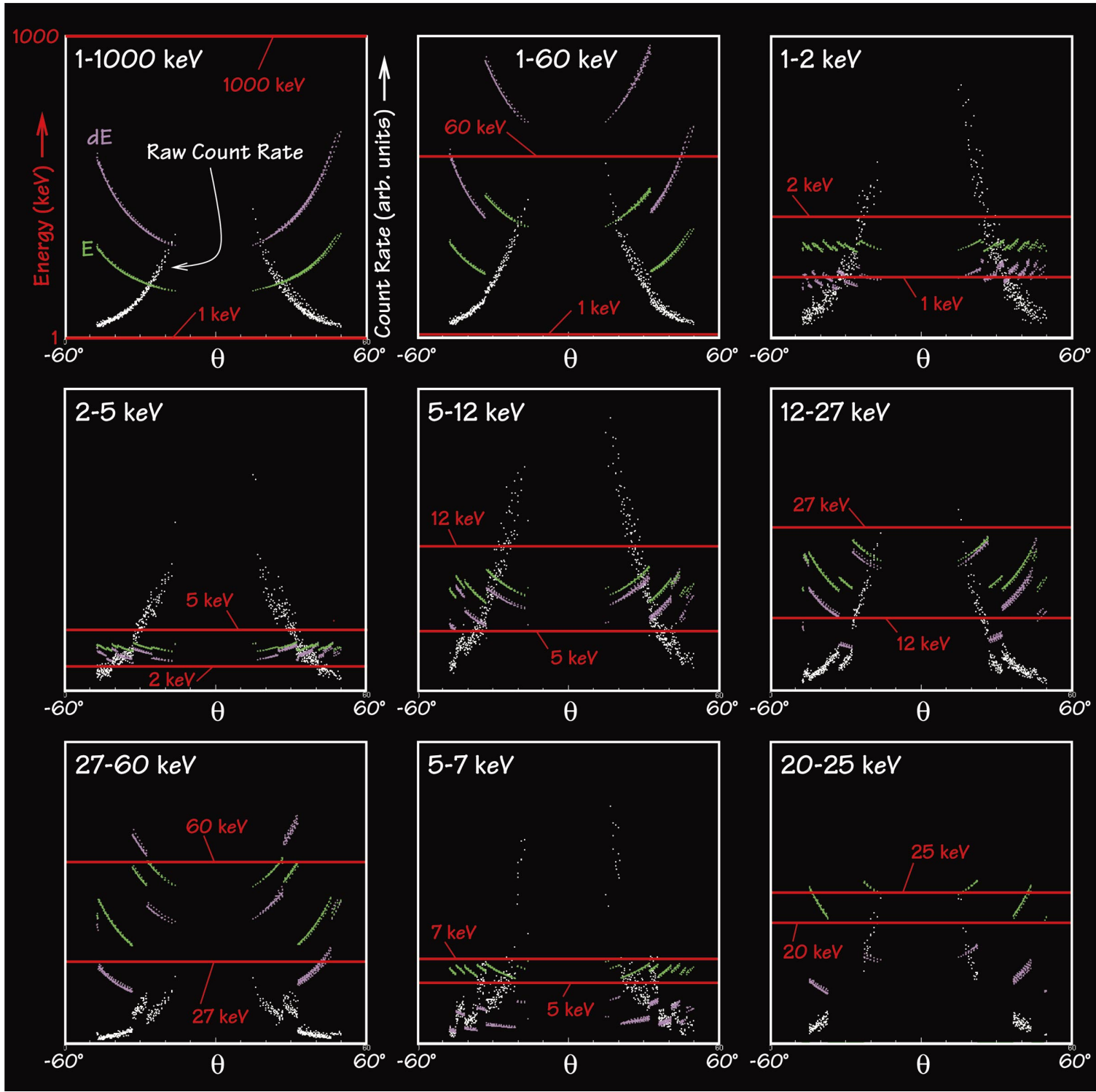

FIG. 15. (Color) Variation of energy and $\Delta E$ as a function of polar angle and requested energy band. For a given energy band, the actual $E_{\mathrm{mid}}$ (green points) and $\Delta E$ (purple points) will vary with polar angle. The white points are the polar angle distribution of the raw counts $(210$ spins of data from October 4,2000$)$. All quantities are plotted in arbitrary units and the scaling is different for all panels. However, to get a sense of the energy scaling, the two horizontal red lines drawn on each panel indicate the requested energy range (e.g., 1-2 keV for the upper right hand panel and 20-25 keV for the lower right hand panel). Note that for all but the 1-1000 keV case, the $E$ and $\Delta E$ "curves" are discontinuous functions of polar angle. This is due to the fact that different groups of time-of-flight-byte bins need to be used at different polar angles. As can be seen in the raw count rate distributions, introduction of discontinuities in the data is the main consequence of this inconsistency. But notice also, that gaps in coverage can occur when $\Delta E / E$ becomes small as is the case for the $20-25 \mathrm{keV}$ panel.

appears in Eq. (43) is not the one explicitly defined by the requested energy band. Rather it is the one implicitly implied by the specific grouping of TOF bytes that actually get used at each polar angle. This is a serious problem because it means that both $\Delta E$ and $E$ vary with polar angle. To illustrate this more clearly, we plot $\Delta E$ (purple points) and $E$ (green points) as a function of polar angle in Fig. 15 together with the raw count rate (white points) as a function of $\theta$. In this plot we have not binned the data into regular polar angle bins which is why there are so many different angles represented.

While the variation of $E$ and $\Delta E$ is apparent in each panel, the most obvious and disturbing feature of this plot is that for all but the 1-1000 keV requested energy band, $E$ and $\Delta E$ are in fact discontinuous functions of polar angle. The effect these discontinuities have on the raw count rate is clearly visible. Note that, even for a requested energy band of 1-60 keV, small but very noticeable discontinuities appear in the count rate data. These artifacts get much more severe for the higher energy bands and/or when the requested $\Delta E$ gets smaller (i.e., when $\Delta E / E$ gets small). Another artifact that is introduced when $\Delta E / E$ becomes too small is that gaps begin to appear in polar angle coverage as shown in the $20-25 \mathrm{keV}$ panel. This occurs because $\Delta E$ gets so small that none of the TOF-byte midpoint energies fall in the requested energy range for some polar angle ranges.

\section{B. Solution to energy binning problems}

At first glance, it may seem obvious that the way to correct for the discontinuities in the count rate data is to 
divide by the appropriate value of $\Delta E$. The reason this does not work is that while it formally results in a proper differential flux, the energy, $E$, at which the differential flux is evaluated for still changes discontinuously as a function of polar angle. In other words, dividing by the proper $\Delta E$ produces differential flux images where each polar angle represents a different energy. In addition, this approach does nothing to avoid the introduction of gaps in polar angle. Clearly, in order to be able to generate uniform-energy differential flux images at arbitrary energies, a different approach is necessary. In this section we describe an alternative processing scheme that overcomes these problems and is also reasonably simple to implement.

Rather than trying to bin the data into pre-selected energy bands (e.g., $1-2 \mathrm{keV}, 2-5 \mathrm{keV}$, etc.), we instead construct a "differential flux versus energy spectrum" for each pixel in the final image. Once this is done, the differential flux at any desired energy can be interpolated from these spectra. This approach ensures that the fluxes in each of the pixels in the final image will be evaluated at the same energy and it also eliminates the gaps that were described in the previous section. The task of creating differential flux images therefore boils down to three distinct subproblems: (1) Calculation of the differential flux energy spectrum for a given pixel in a given head; (2) combining the spectra together in pixels where heads overlap, and; (3) interpolating to the desired energy.

\section{Differential flux energy spectra}

From Eq. (43), we can write

$$
\begin{aligned}
j_{0, i j k \ell t}\left(E_{i j k t}\right) & =\frac{C_{i j k \ell t}}{G_{i j k}\left(E_{i j k t}\right) \Delta E_{i j k t}} \\
& =\frac{C_{i j k \ell t}}{F_{i j k}\left(E_{i j k t}\right)},
\end{aligned}
$$

where, $F_{i j k}\left(E_{i j k t}\right)$ is the "flux conversion factor" (FCF), $i$ is the start-byte index, $j$ is the stop-byte index, $k$ is the head ID, $\ell$ is the azimuth bin number, and $t$ is the TOF-byte index. As we have noted above, this simple formula can be used to convert raw count rate to flux provided that the true flux $j$ and the geometric factor $G$ do not change very much over the range of energies considered, $\Delta E$. In our case this is a reasonable approximation since $G$ is approximately independent of energy above $1 \mathrm{keV}$ and each TOF byte implies a fairly narrow "energy aperture."

Our algorithm for computing the differential flux versus energy spectrum in a single pixel for a single head is outlined below.

- Define the polar angle pixels. Nominally these will be $4^{\circ}$ or $5^{\circ}$ wide, but for a given head they can be any reasonably small value. However, in order to properly merge the final spectra for each head together into a single image, we should restrict the polar angle bin size to values that are multiplicative factors of $20^{\circ}$.

- For a given pixel, scan through the raw direct events and select those that contribute to the pixel, i.e., select those events that have start/stop/azimuth byte combinations that fall in the $\Delta \theta / \Delta \phi$ range implied by the selected pixel.

- Convert each selected event to a differential flux using the appropriate geometric factor, $G_{i j k}$, and the $\Delta E$ implied by its TOF-byte value. Also keep track of all the corresponding energies that result from this operation. At this point we technically have a differential flux versus energy spectrum in the pixel. However, in practice the counting statistics will be extremely poor if we stop here.

- To build up counting statistics, we bin the differential fluxes into a smaller number of discrete TOF bins. One way to accomplish this is to average the fluxes into 64 bins according to their TOF values. The energy that we associate with each bin is then taken to be the average energy of the fluxes that went into that bin. Since there are many TOF bins at the lower energies, each with very narrow $\Delta E$ ranges, the counting statistics at low energies can still be quite poor. Thus an alternate binning scheme in which groups of low-energy TOF bins are averaged together can be used instead. The latter binning scheme generally yields better results.

\section{Combining spectra from different heads}

Once the differential flux versus energy spectrum has been determined for each pixel in all three heads, we are ready to combine the three heads together. In pixels of the final image where two heads overlap (note that there are no pixels for which three heads ever overlap), the two different energy spectra must be merged together. One way to accomplish this is to simply create a new merged spectrum from the flux versus energy data points in each head (this will result in a merged spectrum with twice the data points contained in a single head spectrum). A negative aspect of this approach is that it often introduces artifacts into the final image because the spectrum from one head may have much poorer counting statistics than the other. In order to solve this problem, we use a weighted average of the individual spectra instead of just merging the data points. The weights are determined by the number of counts that went into each flux estimate.

\section{Interpolating to the desired energy}

After the energy spectrum for a given pixel has been computed, the differential flux at a given energy is obtained by interpolation (or extrapolation if necessary). Several interpolation methods have been implemented including: linear interpolation; B-spline interpolation, Cardinal spline interpolation (joined Bezier spline segments in which only zeroeth and first order derivatives are forced to be continuous); robust straight line fitting of nearby points (with an adjustable number of points in the fit); Maxwellian distribution fit; and a Kappa distribution ${ }^{6}$ fit.

The best results are obtained with the B-spline interpolation and the Kappa fit. For the B-spline interpolation the data points (flux versus energy) are taken to be the control points of the B-spline curve. Note that for this type of spline, 


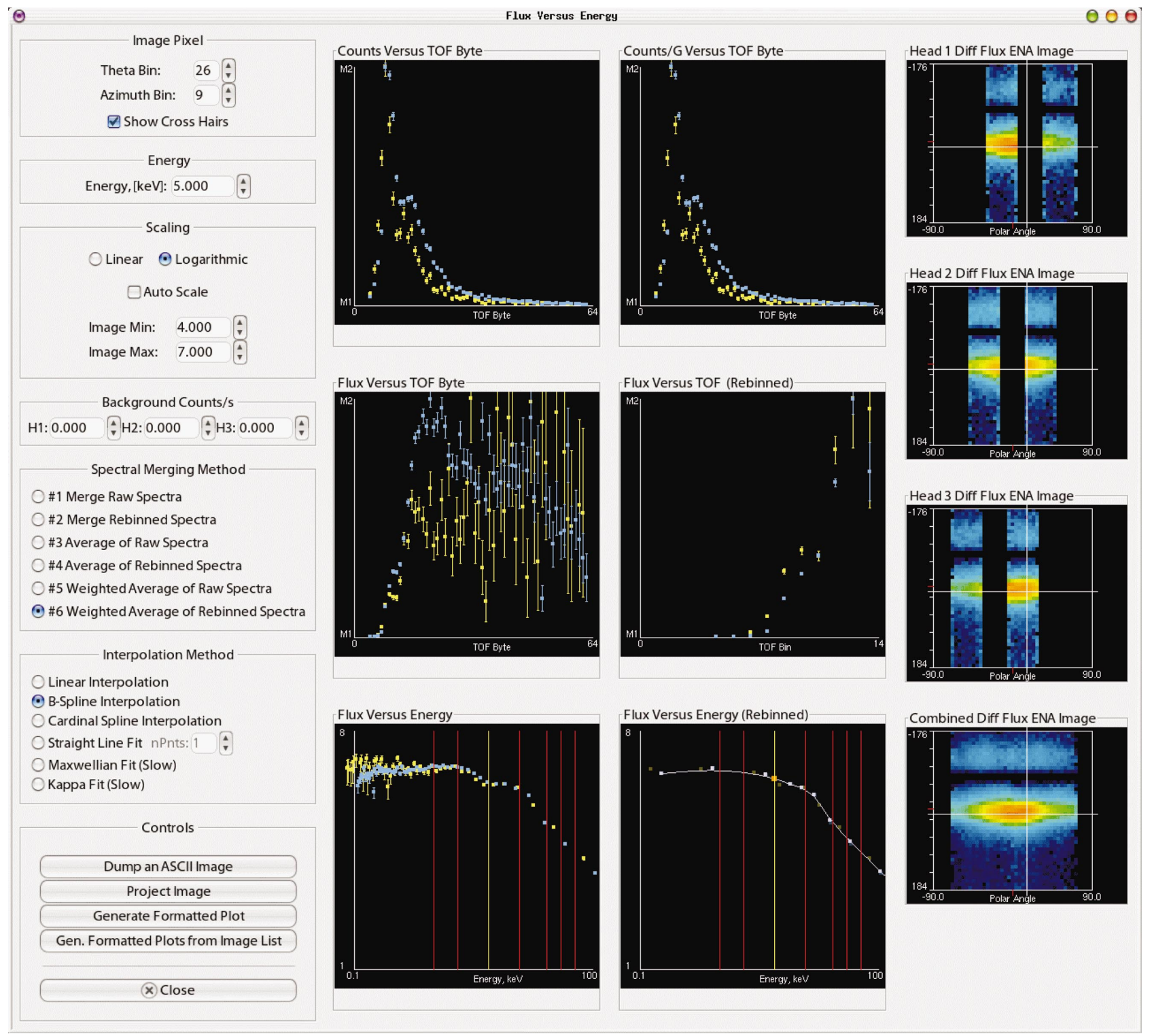

FIG. 16. (Color) Interactive graphical tool illustrating how differential flux images can be created without energy binning.

the interpolated curve always lies in the "convex hull" of the piece-wise linear curve defined by the control points. ${ }^{7}$ Thus, the curve is not forced to go through any of the data points and will never "blow up."

An interactive graphical tool illustrating the steps outlined above is shown in Fig. 16. From left to right and top to bottom, the six panels containing line plots show: Counts versus TOF byte; counts/G versus TOF byte; differential flux versus TOF byte; differential flux versus re-binned TOF bins; differential flux versus energy; differential flux versus energy from re-binned TOF bins. The four panels on the right show the differential flux images obtained from each head plus the combined image. The line plots correspond to the pixel marked with a cross hair. The differential flux images were evaluated at $5 \mathrm{keV}$ and the interpolated value for the pixel under the cross hair is shown as a larger orange square in the lower-right line plot. The vertical lines on the lower two plots are drawn at energies of $(1,2,5,12,27,40$, and $60 \mathrm{keV})$.

The data shown in Fig. 16 were acquired on orbit by
MENA over an accumulation time of 420 min between 17 and 24 UT on October 4, 2000. Although for routine scientific analysis we would not consider using such long integration times, here it is useful to monitor the effects of our processing without the counting noise obscuring the results. Although a few artifacts remain in the final differential flux image (in the form of vertical stripes), the overall quality of the image is quite high. An additional feature that can be seen in the energy spectra for these long-time-average images is the possible presence of two distinct particle populations. This can be seen in the differential flux versus energy plot shown in the lower left hand panel of Fig. 16. It is quite possible that the component appearing at lower energies could be due to oxygen ENAs, although more work needs to be done in order to verify this hypothesis.

A set of differential flux images for a different event are shown in Fig. 17(a). The data for these images were acquired by MENA on June 18, 2001 over the 40 min period from 1550-1630 UT. Differential flux images at 5, 10, 12, 14, 16, 18,20 , and $30 \mathrm{keV}$ are shown. In each image the view is 

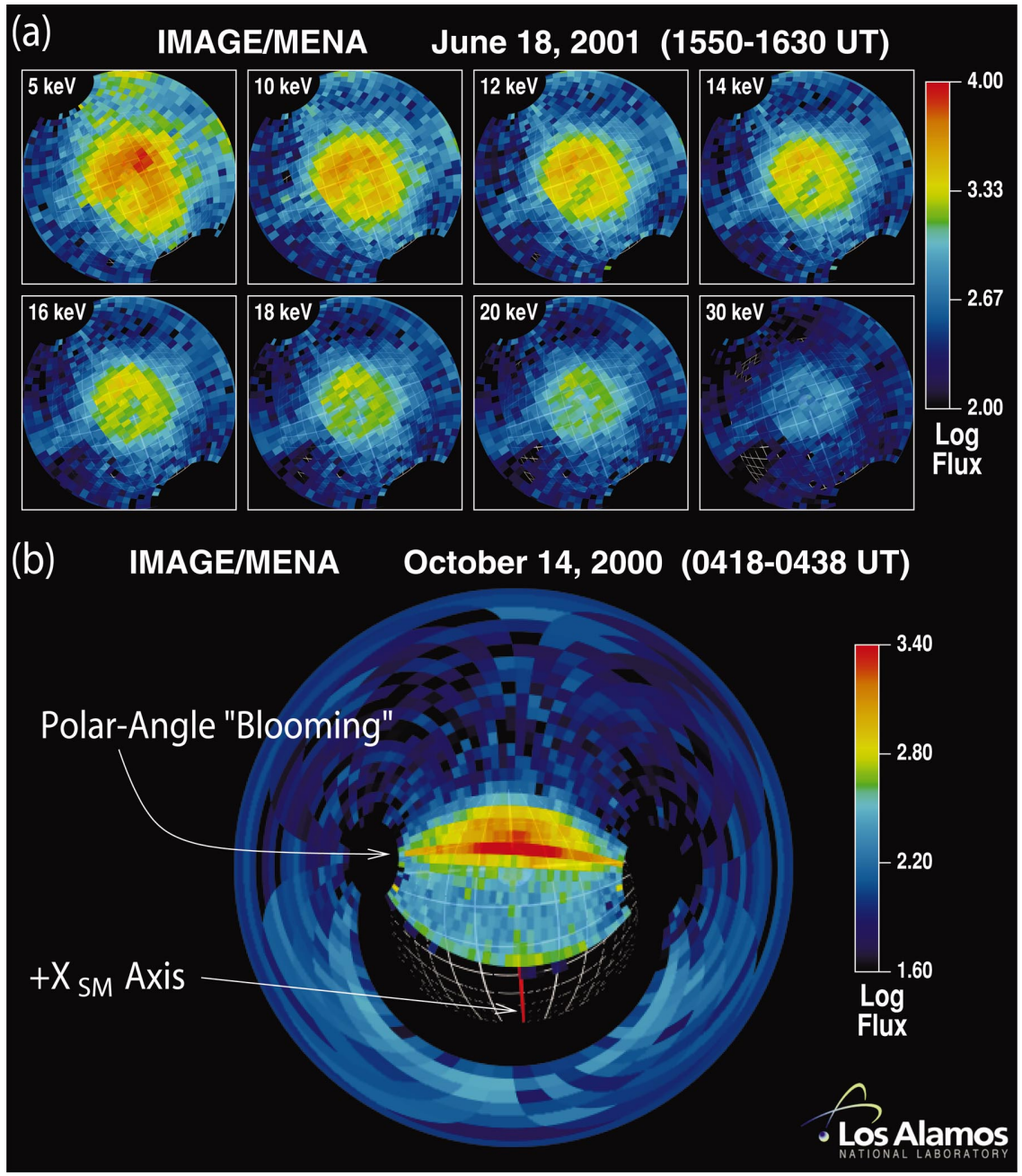

FIG. 17. (Color) (a) Differential flux images at eight different energies constructed from IMAGE/MENA direct-events data acquired during a storm on June 18, 2001. (b) An example of polar-angle "blooming" in an image acquired on October 14, 2000. The units for flux in both panels are number/( $\left.\mathrm{cm}^{2} \cdot \mathrm{sr} \cdot \mathrm{keV} \cdot \mathrm{s}\right)$.

from the northern hemisphere $\left(r_{\mathrm{GSM}}=3.0,0.6,7.5 R_{E}\right)$ looking down toward the center of the earth which is located at the center of each image. The sun (i.e., local magnetic noon) is roughly toward the top (closer to "11 o'clock" position) of each image and local magnetic midnight is roughly toward the bottom (closer to "5 o'clock position"). A magnetic storm was under way during this event and the images reveal a somewhat unusual morphology in which the ring current peaks on the day side. This is consistent with data acquired by the higher-energy ENA imager (HENA) on the IMAGE spacecraft. $^{8}$

\section{OUTSTANDING ISSUES AND FUTURE WORK}

In the previous sections we discussed processing algorithms that we have developed for creating both integral and differential flux images from the raw MENA direct events data and the example shown in Fig. 17(a) clearly demonstrates the effectiveness of our approach. Nevertheless, several outstanding issues remain that we still need to address and we will briefly discuss them below.

\section{A. Scattering, point spread function, and image deconvolution}

In our calculation of the geometric factors above, we assumed that ENAs incident on the start foil [see Fig. 1(d)] pass through it unperturbed on straight-line trajectories. In reality, the thin carbon foils used in the MENA instrument can produce a significant degree of angular scattering of the incident ENAs. As discussed by Funsten, McComas, and Barraclough, ${ }^{9}$ the scattering half angle is inversely proportional to the incident particle energy

$$
\psi_{1 / 2}=\frac{k_{F}}{E},
$$

where the "foil constant," $k_{F}$ depends on the incident particle type (e.g., $\mathrm{H}$ or O), the target composition (carbon in our case), and the thickness of the foil (for MENA, the carbon foils have a mass per area value of approximately $1.1 \mu \mathrm{g} / \mathrm{cm}^{2}$ giving a thickness of $\approx 49 \AA$ ).

The overall effect of the scattering in the carbon foils is to broaden the polar angle profiles in each head. The broadening is worst at the lowest energies and improves substantially at higher energies. In addition, for a given energy, heavier atoms (e.g., He, O, etc.) scatter much more than 
lighter ones do (i.e., H). In order to remove this undesirable effect, the "scattering function" must be deconvolved from the images in the head frame. Although simple Fourier methods for image deconvolution exist, ${ }^{10}$ it is important to recognize that they do not work well in deconvolving extended sources and they also rarely yield acceptable results in situations where there is significant noise present. Several deconvolution methods that are more suitable for our purposes are discussed in Jansson's book ${ }^{11}$ and in a review article by Starck, Pantin, and Murtagh. ${ }^{12}$ The so-called "Damped Richardson-Lucy" algorithm is a particularly appealing candidate because: it is explicitly based on a maximumlikelihood solution for Poisson statistics; it "conserves energy;" it ensures "positivity;" it is iterative and therefore fast; and it is specifically designed to work with extended sources. We have implemented the method and applied it to the final images with some encouraging initial results (but we note that a proper implementation requires the deconvolution to be done in the head frame, not the final image frame).

\section{B. Point spread function and polar-angle "blooming" artifacts}

Perhaps the most obvious and unnatural artifact in the MENA images is the "polar angle blooming" effect as illustrated in Fig. 17(b). It seems to occur when MENA is imaging particularly intense low-altitude emissions from near the limb of the earth at relatively oblique viewing angles (i.e., when IMAGE is not high above the pole). Under these conditions, the azimuth bins affected (usually only one or two of them) show a broad enhancement over an anomalously wide range of polar angles. This feature is indicated by an arrow in Fig. 17(b). Our initial, tentative interpretation of this feature is that it could be a result of the low-altitude ENA emissions having a significant oxygen content. Since oxygen neutral atoms (at a given energy per amu) will undergo substantially more scattering in the thin carbon foil (e.g., see Funsten, McComas, and Barraclough ${ }^{9}$ ), the signal would "spread out" more in the imaging (polar-angle) direction. Note that this implies that the point spread function in the polar angle direction is likely to be species dependent as we mentioned in the previous section.

\section{Sun signal contamination}

Although the nano-structure transmission grating used in the IMAGE/MENA heads was designed to reject UV photons (while at the same time transmitting ENAs), the IMAGE/MENA instrument still turns off once each 2 min spin period as it sweeps past the sun in order to avoid damage to the detectors from the very intense flux of solar UV photons. Despite this safeguard, we have discovered that the MENA instrument still appears to respond to solar photonsand in certain circumstances the peak response can occur when the instrument is not even looking directly at the sun.

In order to determine the source of this contamination, we have computed the total projected (or "effective") area of solar-illuminated surfaces upstream of the gratings (collimator plates and inner surfaces of the collimator housing structure) as a function of spin phase angle. We find that this projected area is highly correlated with the polar-anglesummed count rate versus spin phase. We therefore surmise that the anomalous sun signal present in the data is due to reflection of solar UV photons from the collimator plates and from the collimator housing structure into the detector.

A detailed calculation of these effects from first principles would be extremely difficult and is far beyond the scope of the present article. Although more work needs to be done in modeling the photon response, the removal of this artifact from the images will almost certainly be accomplished with an ad hoc empirical processing scheme.

\section{Correct background subtraction}

In counting experiments, the number of counts observed typically obeys a Poisson distribution. If we have an underlying count rate, $r$, and an observing time, $T$, then the observed number of counts, $n$, will be Poisson-distributed as follows:

$$
p(n \mid r)=\frac{(r T)^{n}}{n !} e^{-r T} .
$$

Here, $p(n \mid r)$ is the probability of getting $n$ counts given that we know that the rate has a value $r$. However, our problem is quite different from this one. We wish to infer the value of $r$, given that we observed $n$ counts in the (known) time interval, $T$. In other words, what we need to compute is $p(r \mid n)$. This can be accomplished by using Bayes' theorem which gives (e.g., see Loredo ${ }^{13}$ and D'Agostini ${ }^{14}$ ) the "posterior distribution"

$$
p(r \mid n)=\frac{T(r T)^{n} e^{-r T}}{n !} .
$$

The mode (most probable value, i.e., the peak of the distribution), mean $\left(\langle r\rangle=\int r p(r \mid n) d r\right)$, and standard deviation $\left(\left\langle r^{2}\right\rangle-\langle r\rangle^{2}\right)^{1 / 2}$ of this distribution are: $n / T,(n+1) / T$, and $\sqrt{n+1} / T$, respectively. One way to summarize the inferred value of $r$ is to report the most probable value (the mode) together with a "credibility region" containing $95 \%$ of the posterior density. The credibility region will in general be asymmetric about the mode and will never contain negative numbers. Note that when $n$ is large, the Poisson distribution can be approximated by a Gaussian with mode, mean, and standard deviation of: $n / T, n / T$, and $\sqrt{n} / T$, respectively, which is consistent with the Bayesian result in the limit of large $n$.

Although this result seems trivial (we would have chosen $r=n / T$ even without the Bayesian analysis), it does allow a more rigorous treatment of uncertainties and, as we shall see, the Bayesian approach allows us to correctly deal with background subtraction. The following description of correct background subtraction is discussed much more thoroughly in Loredo. ${ }^{13}$

If we have a signal with rate $s$ and a background with rate $b$ and we do not know what either of these values are, then they have to be inferred from the observed count rates. One way to do this is to measure a count rate while looking at the "signal+background" and also to measure the count rate while looking away from the signal (where presumably 
we only have background). If we collect $N_{\text {on }}$ counts in a time $T_{\text {on }}$ when we are looking at the signal+background and we collect $N_{\text {off }}$ counts in a time $T_{\text {off }}$ when we are looking at just the background, then it would be tempting to compute $s$ $=N_{\text {on }} / T_{\text {on }}-N_{\text {off }} / T_{\text {off }}$ as the "background-subtracted" signal. Unfortunately, this approach is incorrect despite the fact that, in practice, it is the method employed almost universally. The most obvious symptom that it is an incorrect prescription for background subtraction is that the resulting signals can be negative-a result that we know a priori can never happen.

The correct approach to obtaining the signal $s$ is via Bayes' theorem. As described in detail by Loredo, ${ }^{13}$ application of Bayes' theorem to this problem leads to the following posterior for $s \geqslant 0$ :

$$
p\left(s \mid N_{\text {on }} I\right)=\sum_{1=0}^{N_{\text {on }}} C_{i} \frac{T_{\text {on }}\left(s T_{\text {on }}\right)^{i} e^{-s T_{\text {on }}}}{i !}
$$

where

$$
C_{i}=\frac{\left(1+\frac{T_{\mathrm{off}}}{T_{\mathrm{on}}}\right)^{i} \frac{\left(N_{\mathrm{on}}+N_{\mathrm{off}}-i\right) !}{\left(N_{\mathrm{on}}-i\right) !}}{\sum_{j=0}^{N_{\mathrm{on}}}\left(1+\frac{T_{\mathrm{off}}}{T_{\mathrm{on}}}\right)^{j} \frac{\left(N_{\mathrm{on}}+N_{\mathrm{off}}-j\right) !}{\left(N_{\mathrm{on}}-j\right) !}} .
$$

Application of this approach to the IMAGE/MENA data may result in qualitatively "better looking" background subtracted images. And it may also be quantitatively important in ENA inversion schemes as well as the possible extraction of weak signals such as one might expect from the heliospheric ENA emissions or weak down-tail magnetospheric emissions.

\section{E. MCP design considerations}

As we have seen, the inherent mismatch between the requested $\Delta E$ and the actual $\Delta E$ combined with the polar- angle-dependent TOF byte to energy mapping, results in severe artifacts in the differential flux images. We note that this would not have occurred if the TOF-byte-to-energy mappings were fixed (i.e., not angle dependent)—we would then just use the "natural" $E$ and $\Delta E$ values implied by the appropriate TOF-byte groupings. This could have been achieved with a curved MCP/anode detector since the path length would then be constant. Thus, it may be appropriate to consider adopting a cylindrically curved MCP stack in future versions of the MENA instrument.

${ }^{1}$ C. J. Pollock, K. Asamura, J. Baldonado, M. M. Balkey, P. Barker, J. L. Burch, E. J. Korpela, J. Cravens, G. Dirks, and M. C. Fok et al., Space Sci. Rev. 91, 113 (2000).

${ }^{2}$ J. D. Sullivan, Nucl. Instrum. Methods 95, 5 (1971).

${ }^{3}$ M. G. Tuszewski, T. E. Cayton, and J. C. Ingraham, Nucl. Instrum. Methods Phys. Res. A 482, 653 (2002).

${ }^{4}$ J. B. Blake, J. F. Fennell, L. M. Friesen, B. H. Johnson, W. A. Kolasinski, D. J. Mabry, J. V. Osborn, S. H. Penzin, E. R. Schnauss, and H. E. Spence et al., Space Sci. Rev. 71, 531 (1995).

${ }^{5}$ J. F. Ziegler, P. B., and U. Littmark, The Stopping and Range of Ions in Solids (Pergamon, New York, 1985).

${ }^{6}$ V. M. Vasyliunas, J. Geophys. Res. 73, 2839 (1968).

${ }^{7}$ J. D. Foley, A. van Dam, S. K. Feiner, and J. F. Hughes, Computer Graphics: Principles and Practice (Addison-Wesley, New York, 1990).

${ }^{8}$ J. D. Perez, X.-X. Zhang, P. C. Brandt, D. G. Mitchell, J.-M. Jahn, C. J. Pollock, and S. B. Mende, J. Geophys. Res. 109, A09202 (2004).

${ }^{9}$ H. O. Funsten, D. J. McComas, and B. L. Barraclough, Opt. Eng. (Bellingham) 32, 3090 (1993).

${ }^{10}$ W. H. Press, A. A. Teukolsky, W. T. Vetterling, and B. P. Flannery, $\mathrm{Nu}-$ merical Recipes in C (Cambridge University Press, New York, 1992).

${ }^{11}$ P. A. Jansson, Deconvolution of Images and Spectra (Academic, San Diego, 1997).

${ }^{12}$ J. L. Starck, E. Pantin, and F. Murtagh, Publ. Astron. Soc. Pac. 114, 1051 (2002).

${ }^{13}$ T. J. Loredo, in Statistical Challenges in Modern Astronomy, edited by E. D. Feigelson and G. J. Babu (Springer, New York, 1992), pp. 275-297.

${ }^{14}$ G. D'Agostini, Bayesian Reasoning in Data Analysis: A Critical Introduction (World Scientific, Singapore, 2003). 